\title{
Sparticle mass hierarchies, simplified models from SUGRA unification, and benchmarks for LHC Run-II SUSY searches
}

\author{
David Francescone, ${ }^{a}$ Sujeet Akula, ${ }^{b, c}$ Baris Altunkaynak ${ }^{d}$ and Pran Nath ${ }^{a}$ \\ ${ }^{a}$ Department of Physics, Northeastern University, \\ Boston, MA, 02115 U.S.A. \\ ${ }^{b}$ MTA-DE Particle Physics Research Group, University of Debrecen, \\ P.O. Box 105, Debrecen, H-4010 Hungary \\ ${ }^{c}$ ARC Centre of Excellence for Particle Physics at the Terascale, \\ School of Physics, Monash University, \\ Melbourne Victoria, 3800 Australia \\ ${ }^{d}$ Homer L. Dodge Department of Physics and Astronomy, University of Oklahoma, \\ Norman, OK, 73019 U.S.A. \\ E-mail: d.francescone@neu.edu, sujeet.akula@coepp.org.au, \\ baris.altunkaynak@ou.edu, p.nath@neu.edu
}

ABSTRACT: Sparticle mass hierarchies contain significant information regarding the origin and nature of supersymmetry breaking. The hierarchical patterns are severely constrained by electroweak symmetry breaking as well as by the astrophysical and particle physics data. They are further constrained by the Higgs boson mass measurement. The sparticle mass hierarchies can be used to generate simplified models consistent with the high scale models. In this work we consider supergravity models with universal boundary conditions for soft parameters at the unification scale as well as supergravity models with nonuniversalities and delineate the list of sparticle mass hierarchies for the five lightest sparticles. Simplified models can be obtained by a truncation of these, retaining a smaller set of lightest particles. The mass hierarchies and their truncated versions enlarge significantly the list of simplified models currently being used in the literature. Benchmarks for a variety of supergravity unified models appropriate for SUSY searches at future colliders are also presented. The signature analysis of two benchmark models has been carried out and a discussion of the searches needed for their discovery at LHC Run-II is given. An analysis of the spinindependent neutralino-proton cross section exhibiting the Higgs boson mass dependence and the hierarchical patterns is also carried out. It is seen that a knowledge of the spinindependent neutralino-proton cross section and the neutralino mass will narrow down the 
list of the allowed sparticle mass hierarchies. Thus dark matter experiments along with analyses for the LHC Run-II will provide strong clues to the nature of symmetry breaking at the unification scale.

KEYwORDS: Supersymmetry Phenomenology

ArXiv EPRINT: 1410.4999 


\section{Contents}

1 Introduction 1

2 Details of the analysis 3

3 Sparticle mass hierarchies and simplified models 10

4 Model signatures $\quad 13$

5 Benchmarks for future SUSY searches at colliders $\quad 16$

$\begin{array}{ll}5.1 \text { Signature analysis for an mSUGRA benchmark } & 16\end{array}$

$\begin{array}{ll}5.2 \text { Signature analysis for an nuSUGRA benchmark } & 17\end{array}$

$\begin{array}{lll}6 & \text { Dark matter } & 19\end{array}$

$\begin{array}{lll}7 & \text { Conclusion } & 24\end{array}$

\section{Introduction}

The discovery [1, 2] of the Higgs boson [3-5] and the measurement of its mass at $\sim 126 \mathrm{GeV}$ have strong implications for discovery of supersymmetry. In the minimal supersymmetric standard model (MSSM) one identifies the observed Higgs boson as the lightest $C P$-even state $h^{0}$ (see e.g., [6-13]). It is noteworthy that the observed Higgs boson mass lies below but close to the upper limit on the Higgs boson mass predicted in supergravity grand unified models [14-17] with radiative breaking of the electroweak symmetry (for a review see [18]), and this upper limit is well known to be around $130 \mathrm{GeV}[6-13,19,20]$. Further, in supersymmetric models and specifically those within supergravity grand unification, one finds that a Higgs mass of $\sim 126 \mathrm{GeV}$ implies the scale of supersymmetry to be large with the squark masses typically lying in the few $\mathrm{TeV}$ region [6-13, 19-21]. The largeness of the SUSY scale explains the non-observation of sparticles in searches in Run-I of the LHC. However, the LHC energy is being ramped up to $\sqrt{s}=13 \mathrm{TeV}$ and one expects some of the light sparticles to show up in Run-II. ${ }^{1}$

The nature of the observed sparticles, and more generally the hierarchical mass patterns, hold a key to the nature of symmetry breaking at high scales in unified models. Given that there are 31 additional particles beyond the spectrum of the Standard Model, there are a priori $31 ! \sim 8 \times 10^{33}$ ways in which these particles can arrange themselves.

\footnotetext{
${ }^{1}$ Although the current plan is for the LHC to operate at $\sqrt{s}=13 \mathrm{TeV}$ for Run-II, we will carry out the analysis at $\sqrt{s}=14 \mathrm{TeV}$, using the Snowmass [22] Standard Model backgrounds.
} 
This is the landscape of possible mass hierarchies of the new particles. ${ }^{2,3}$ The number of allowed possibilities is significantly reduced in supergravity grand unification with radiative breaking of the electroweak symmetry [23-25]. Additionally, the accelerator and dark matter constraints further reduce the allowed number of possibilities. The landscape of supergravity based models was analyzed in a number of works [23-30] which, however, were all before the discovery of the Higgs boson and a measurement of its mass.

Regarding the Higgs boson mass of $126 \mathrm{GeV}$, there are a limited number of ways in which one can lift its tree mass which lies below $M_{Z}$ to the observed value. These include $D$-term contributions from extra U(1)'s, loop contributions from extra matter [31] or large loop corrections from within the MSSM. The latter possibility implies a relatively high scale of supersymmetry, which explains in part the reason for its non-observation thus far. In this work, we revisit the sparticle landscape analysis taking into account the constraint from the Higgs boson mass measurement on the sparticle landscape. We analyze several different classes of high scale models: these include mSUGRA (also called CMSSM) and supergravity models with nonuniversal boundary conditions at the grand unification scale which include nonuniversalities in the $\mathrm{SU}(2)_{L}$ and $\mathrm{SU}(3)_{C}$ gaugino sector, in the Higgs sector and in the third generation sfermion sector. The most dominant hierarchical patterns that emerge are identified. The hierarchical patterns provide a simple way to connect the simplified models [32-40] with grand unified models. Specifically, we consider five particle mass hierarchies where the various combinations of the five lightest particles that originate in supergravity models are investigated. These five particle mass hierarchies effectively constitute existing and novel simplified models. The hierarchy of five particles can be further truncated to give simplified models with three or four lightest particles as has been more common. It should be noted that these simplified models are obviously part of a UV complete theory since they are obtained by truncation of the spectrum arising from a high scale model.

The outline of the rest of the paper is as follows: in section 2 we give details of the analysis and a cartography of the allowed 5 particle mass hierarchies including the LSP. Five different classes of high scale boundary conditions are analyzed. In section 3 we connect simplified models to the sparticle mass hierarchies. In section 4 we discuss the generic signatures that one expects for the mass patterns. In section 5 we give benchmarks for future searches for supersymmetry at colliders. We also give a signature analysis of two benchmark models with a discussion of cuts needed for their discovery at the LHC Run-II. In section 6 we carry out an analysis of spin independent neutralino-proton cross section in terms of the sparticle mass patterns to see how a measurement of the spin-independent cross section along with a knowledge of the neutralino mass can narrow down the possible hierarchical patterns. Conclusions are given in section 7 .

\footnotetext{
${ }^{2}$ We would loosely call these the sparticle mass hierarchies even though they contain the Higgs boson states, $H^{0}, A^{0}, H^{ \pm}$, which are $R$ parity even.

${ }^{3}$ The landscape is even larger in that the mass gaps among the sparticles can vary continuously which makes the allowed sparticle landscape larger than even the string landscape which has as many as $10^{500}$ possible vacua.
} 


\section{Details of the analysis}

We consider several different supergravity models of soft breaking. These include supergravity models with (1) universal boundary conditions as well as supergravity models with nonuniversal boundary conditions (nuSUGRA) at the grand unification scale. ${ }^{4}$ Within nuSUGRA, we consider (2) nonuniversalities in the $\mathrm{SU}(2)_{L}$ gaugino mass sector, (3) nonuniversalities in the $\mathrm{SU}(3)_{C}$ gaugino mass sector, (4) nonuniversalities in the Higgs sector, and (5) nonuniversalities in the flavor sector with the sfermion masses for the third generation being different from the masses in the first two generations.

Model [1]: mSUGRA:

$$
m_{0} \in[0.1,10] \mathrm{TeV}, \quad m_{1 / 2} \in[0.1,1.5] \mathrm{TeV}, \quad \frac{A_{0}}{m_{0}} \in[-5,5], \quad \tan \beta \in[2,50] .
$$

Model [2]: nuSUGRA, light chargino:

$$
\begin{aligned}
& m_{0} \in[0.1,10] \mathrm{TeV}, \quad M_{1}=M_{3}=m_{1 / 2} \in[0.1,1.5] \mathrm{TeV}, \quad M_{2}=\alpha m_{1 / 2}, \quad \alpha \in\left[\frac{1}{2}, 1\right], \\
& \frac{A_{0}}{m_{0}} \in[-5,5], \quad \tan \beta \in[2,50] .
\end{aligned}
$$

Model [3]: nuSUGRA, light gluino:

$$
\begin{aligned}
& m_{0} \in[0.1,10] \mathrm{TeV}, \quad M_{1}=M_{2}=m_{1 / 2} \in[0.1,1.5] \mathrm{TeV}, \quad M_{3}=\alpha m_{1 / 2}, \quad \alpha \in\left[\frac{1}{6}, 1\right], \\
& \frac{A_{0}}{m_{0}} \in[-5,5], \quad \tan \beta \in[2,50] .
\end{aligned}
$$

Model [4]: nuSUGRA, nonuniversal Higgs:

$$
\begin{aligned}
m_{0} & \in[0.1,10] \mathrm{TeV}, \quad m_{1 / 2} \in[0.1,1.5] \mathrm{TeV}, \quad \frac{A_{0}}{m_{0}} \in[-5,5], \quad \tan \beta \in[2,50], \\
m_{H_{i}}\left(\mathrm{M}_{G}\right) & =m_{0}\left(1+\delta_{i}\right), \quad \text { where } \delta_{i} \in[-0.9,1] .
\end{aligned}
$$

Model [5]: nuSUGRA, light 3rd generation:

$$
\begin{aligned}
m_{0}^{(1)} & =m_{0}^{(2)}=m_{0} \in[0.1,10] \mathrm{TeV}, & m_{0}^{(3)} & =\frac{m_{0}}{1 \mathrm{TeV}+m_{0}}, \quad m_{1 / 2} \in[0.1,1.5] \mathrm{TeV}, \\
\frac{A_{0}}{m_{0}} & \in[-5,5], & \tan \beta & \in[2,50] .
\end{aligned}
$$

In each of the above cases, $\mu$ was taken to be positive. The overview of the parameter scan for the five classes of models listed above is given in table 1 . The scans were performed using SUsyKit [54] which employs SOFTSUSY [55] for 2-loop RG evolution including sparticle thresholds and for sparticle mass calculations, FeynHigGs $[56,57]$ for computing the Higgs boson masses, including the recently added resummation to all orders of leading and subleading logs of type $\log \left(m_{\tilde{t}} / m_{t}\right)$ [58], and MICROMEGAs [59] to calculate the relic density and flavor observables. We only considered points where the LSP is the neutralino, the thermal relic density of the LSP is not overabundant, i.e., $\Omega_{\chi} h^{2}<0.12$, and the Higgs boson mass

\footnotetext{
${ }^{4}$ The literature on supergravity models with nonuniversalities is vast. For a sample of works on nonuniversalities the reader may refer to [41-53] and for a review see [30].
} 


\begin{tabular}{|l|c|c|c|c|}
\hline \multicolumn{1}{|c|}{ SUGRA Model } & $\begin{array}{c}\text { Parameter } \\
\text { Points }\end{array}$ & $\begin{array}{c}\text { NLSP } \\
\text { Patterns }\end{array}$ & $\begin{array}{c}\text { 4-sparticle } \\
\text { Patterns }\end{array}$ & $\begin{array}{c}\text { 5-sparticle } \\
\text { Patterns }\end{array}$ \\
\hline [1] mSUGRA & 5453 & 5 & 15 & 32 \\
[2] Light Chargino & 8000 & 4 & 26 & 89 \\
[3] Light Gluino & 8008 & 7 & 44 & 85 \\
[4] Nonuniversal Higgs & 4738 & 5 & 26 & 59 \\
[5] Light 3rd Generation & 2668 & 6 & 26 & 43 \\
\hline
\end{tabular}

Table 1. An overview of the parameter scans, detailing the number of parameter points simulated, the number of NLSP patterns, the number of 4-sparticle and the number of 5-sparticle mass hierarchies for Models [1]-[5].

\begin{tabular}{|c|c|c|c|}
\hline \multicolumn{2}{|c|}{ Model Parameters } & \multicolumn{2}{|c|}{ Observables } \\
\hline$m_{0}$ & $6183 \mathrm{GeV}$ & $m_{h^{0}}$ & $126.1 \mathrm{GeV}$ \\
$m_{1 / 2}$ & $470 \mathrm{GeV}$ & $\left(m_{\widetilde{g}}, m_{\tilde{t}_{1}}\right)$ & $(1257,3601) \mathrm{GeV}$ \\
$A_{0}$ & $-4469 \mathrm{GeV}$ & $\left(m_{\chi_{1}^{0}}, m_{\chi_{2}^{0}}, m_{\chi_{1}^{ \pm}}\right)$ & $(199,271,261) \mathrm{GeV}$ \\
$\tan \beta$ & 52.1 & $\Omega_{\chi_{1}^{0}} h^{2}$ & $<0.12$ \\
$\operatorname{sgn}(\mu)$ & +1 & $\sigma\left(p p \rightarrow \chi_{2}^{0} \chi_{1}^{ \pm}\right)$ & $134.7 \mathrm{fb}$ \\
\hline
\end{tabular}

Table 2. Model parameters and observables of our mSUGRA benchmark model.

\begin{tabular}{|c|c|c|c|}
\hline \multicolumn{2}{|c|}{ Model Parameters } & \multicolumn{2}{|c|}{ Observables } \\
\hline$m_{0}$ & $4 \mathrm{TeV}$ & $m_{h^{0}}$ & $124.7 \mathrm{GeV}$ \\
$\left(M_{1}, M_{2}, M_{3}\right)$ & $(980,520,550) \mathrm{GeV}$ & $\left(m_{\widetilde{g}}, m_{\widetilde{t}_{1}}\right)$ & $(1446,1481) \mathrm{GeV}$ \\
$A_{0}$ & $-7 \mathrm{TeV}$ & $\left(m_{\chi_{1}^{0}}, m_{\chi_{2}^{0}}, m_{\chi_{1}^{ \pm}}\right)$ & $(441,461,462) \mathrm{GeV}$ \\
$\tan \beta$ & 30 & $\Omega_{\chi_{1}^{0}} h^{2}$ & 0.12 \\
$\operatorname{sgn}(\mu)$ & +1 & $\sigma(p p \rightarrow \widetilde{g} \tilde{g})$ & $23.0 \mathrm{fb}$ \\
\hline
\end{tabular}

Table 3. Model parameters and observables of our nuSUGRA benchmark.

is not too light with $m_{h^{0}}>120 \mathrm{GeV} .{ }^{5}$ (For some recent works on mSUGRA see [60-63]). These constraints immediately reduce the available number of sparticle mass hierarchies. Of the large number of model points in the scan of table 1 two models were investigated in detail for their discovery potential at LHC Run-II. One of these is an mSUGRA model whose parameters are listed in table 2 and the other is an nuSUGRA model whose parameters are listed in table 3 . The signatures for these models were investigated using the ATLAS cuts at $\sqrt{s}=8 \mathrm{TeV}$ as given in table 4 .

\footnotetext{
${ }^{5}$ We have chosen a bit generous window on the Higgs boson mass for the following reason: aside from the possible errors in theory computations which are now reduced since we use FeynHiggs, in extended models with extra $\mathrm{U}(1)$ corrections to the Higgs mass can arise of the size of $\mathcal{O}(1 \mathrm{GeV})$ from extra D-term contributions. Also extra vector-like matter if it exists could make a contribution of the same size (see, e.g., [31]). So in order that our analysis also applies to such models, we consider a bit generous window but the results for the narrower window on the Higgs mass are easily extracted from the analysis presented in the paper. For this reason we have color-coded figure 7 for the mSUGRA case and figure 8 for the nuSUGRA cases with the Higgs mass.
} 


\begin{tabular}{|l|c|c|c|c|}
\hline Signal Region & $\mathrm{N}$ jets $\left(p_{\mathrm{T}}\right)$ & $E_{\mathrm{T}}^{\mathrm{miss}}$ & $m_{\mathrm{eff}}$ & $E_{\mathrm{T}}^{\mathrm{miss}} / \sqrt{H_{T}^{6 j}}$ \\
\hline SR- $\ell \ell-4 j-\mathrm{A}$ & $\geq 4(50)$ & $>250$ & $>1300$ & - \\
\hline SR- $\ell-4 j-\mathrm{B}$ & $\geq 4(50)$ & $>350$ & $>1100$ & - \\
\hline SR- $\ell \ell-4 j-\mathrm{C}$ & $\geq 4(30)$ & $>400$ & $>1000$ & $>16$ \\
\hline \hline Signal Region & $\mathrm{N}$ jets $\left(p_{\mathrm{T}}\right)$ & $E_{\mathrm{T}}^{\mathrm{miss}}$ & $m_{\mathrm{eff}}^{\mathrm{inc}}$ & $E_{\mathrm{T}}^{\mathrm{miss}} / \sqrt{H_{T}^{6 j}}$ \\
\hline SR- $\ell \ell-7 j-\mathrm{A}$ & $\geq 7(30)$ & $>200$ & $>1000$ & - \\
\hline SR- $\ell \ell-7 j-\mathrm{B}$ & $\geq 7(30)$ & $>350$ & $>1000$ & - \\
\hline SR- $\ell \ell-7 j-\mathrm{C}$ & $\geq 7(30)$ & $>250$ & $>1500$ & - \\
\hline \hline Signal Region & $\mathrm{N}$ jets $\left(p_{\mathrm{T}}\right)$ & $E_{\mathrm{T}}^{\mathrm{miss}}$ & $m_{\mathrm{T}}$ & $m_{\mathrm{eff}}^{\mathrm{inc}}$ \\
\hline SR- $1 \ell-6 j-\mathrm{A}$ & $\geq 6(30)$ & $>175$ & $>140$ & $>700$ \\
\hline SR- $1 \ell-6 j-\mathrm{B}$ & $\geq 6(30)$ & $>225$ & $>140$ & $>800$ \\
\hline SR- $1 \ell-6 j-\mathrm{C}$ & $\geq 6(30)$ & $>275$ & $>160$ & $>900$ \\
\hline
\end{tabular}

Table 4. A summary of signal regions (SR) with cuts used in the signature analysis of the model benchmark. The top two blocks in the table have the additional constraints of $p_{\mathrm{T}}\left(j_{1}\right)>90 \mathrm{GeV}$, $E_{\mathrm{T}}^{\mathrm{miss}}>150 \mathrm{GeV}, \geq 4$ jets with $p_{\mathrm{T}}>30 \mathrm{GeV}, \Delta \phi_{\text {min }}^{4 j}>0.5, E_{\mathrm{T}}^{\mathrm{miss}} / m_{\mathrm{eff}}^{4 j}>0.2, \geq 3$ b-jets with $p_{\mathrm{T}}>30 \mathrm{GeV}$. The bottom block in the table has the constraints $p_{\mathrm{T}}\left(j_{1}\right)>90 \mathrm{GeV}, E_{\mathrm{T}}^{\text {miss }}>150$, $\geq 4$ jets with $p_{\mathrm{T}}>30 \mathrm{GeV}, \geq 3$ b-jets with $p_{\mathrm{T}}>30 \mathrm{GeV}$. These constraints are adopted from the ATLAS analysis at $\sqrt{s}=8 \mathrm{TeV}[81]$ and applied to our analysis at $\sqrt{s}=14 \mathrm{TeV}$.

We begin the study of the hierarchies by first identifying the next-to-lightest sparticle (NLSP). For each NLSP case, we next determine the possible 4-sparticle hierarchies (LSP, NLSP, and two heavier sparticles). Within these, we additionally identify the next lightest sparticle, giving a hierarchy of the 5 lightest sparticles. These hierarchies are labeled by the following scheme: we begin with a symbol for the NLSP, followed by a number for one of the possible 4-sparticle hierarchies for the given NLSP, and lastly append a letter for the $5^{\text {th }}$ lightest sparticle. We note that when the mass gap $\Delta m\left(H^{0}, A^{0}\right)$ is $\mathcal{O}(1 \mathrm{GeV})$, $H^{0}$ and $A^{0}$ are treated as degenerate in mass in the classification scheme. In table 5 the hierarchical mass patterns for the mSUGRA case are exhibited. In all cases the exhibited mass hierarchies are those that remain after all the constraints have been imposed.

The sparticle mass hierarchies in the nuSUGRA Models [2]-[5] are exhibited in tables 6 to 9. The sparticle mass hierarchies for nuSUGRA Model [2] with nonuniversalities in the $\mathrm{SU}(2)_{L}$ gaugino mass sector are given in table 6, while those for the nuSUGRA Model [3] with nonuniversality in the gluino mass sector are given in table 7 . In table 8 we give an 


\begin{tabular}{|l|l|c|}
\hline Pattern Label & \multicolumn{1}{|c|}{ Mass Hierarchy } & $\%$ \\
\hline $\mathrm{mSP}[\mathrm{C} 1 \mathrm{a}]$ & $\chi_{1}^{ \pm}<\chi_{2}^{0}<\chi_{3}^{0}<\chi_{4}^{0}$ & 83.8 \\
$\mathrm{mSP}[\mathrm{C} 1 \mathrm{~b}]$ & $\chi_{1}^{ \pm}<\chi_{2}^{0}<\chi_{3}^{0}<H^{0}$ & 2.49 \\
$\mathrm{mSP}[\mathrm{C} 1 \mathrm{c}]$ & $\chi_{1}^{ \pm}<\chi_{2}^{0}<\chi_{3}^{0}<\chi_{2}^{ \pm}$ & 1.62 \\
\hline $\mathrm{mSP}[\mathrm{C} 2]$ & $\chi_{1}^{ \pm}<\chi_{2}^{0}<H^{0}<A^{0}$ & 0.65 \\
\hline $\mathrm{mSP}[\mathrm{C} 3]$ & $\chi_{1}^{ \pm}<\chi_{2}^{0}<g<\chi_{3}^{0}$ & 0.04 \\
\hline $\mathrm{mSP}[\mathrm{C} 4]$ & $\chi_{1}^{ \pm}<\chi_{2}^{0}<A^{0}<H^{0}$ & 0.02 \\
\hline $\mathrm{mSP}[\tau 1 \mathrm{a}]$ & $\tau_{1}<\chi_{2}^{0}<\chi_{1}^{ \pm}<H^{0}$ & 3.89 \\
$\mathrm{mSP}[\tau 1 \mathrm{~b}]$ & $\tau_{1}<\chi_{2}^{0}<\chi_{1}^{ \pm}<\mu_{r}$ & 0.89 \\
$\mathrm{mSP}[\tau 1 \mathrm{c}]$ & $\tau_{1}<\chi_{2}^{0}<\chi_{1}^{ \pm}<\nu_{\tau}$ & 0.15 \\
$\mathrm{mSP}[\tau 1 \mathrm{~d}]$ & $\tau_{1}<\chi_{2}^{0}<\chi_{1}^{ \pm}<t_{1}$ & 0.09 \\
\hline $\mathrm{mSP}[\tau 2 \mathrm{a}]$ & $\tau_{1}<\mu_{r}<e_{r}<\chi_{2}^{0}$ & 0.69 \\
$\mathrm{mSP}[\tau 2 \mathrm{~b}]$ & $\tau_{1}<\mu_{r}<e_{r}<\nu_{\tau}$ & 0.52 \\
\hline $\mathrm{mSP}[\tau 3 \mathrm{a}]$ & $\tau_{1}<H^{0}<A^{0}<\chi_{2}^{0}$ & 0.04 \\
$\mathrm{mSP}[\tau 3 \mathrm{~b}]$ & $\tau_{1}<H^{0}<A^{0}<H^{ \pm}$ & 0.02 \\
\hline $\mathrm{mSP}[\tau 4]$ & $\tau_{1}<t_{1}<\chi_{2}^{0}<\chi_{1}^{ \pm}$ & 0.04 \\
\hline $\mathrm{mSP}[\mathrm{t} 1 \mathrm{a}]$ & $t_{1}<\chi_{2}^{0}<\chi_{1}^{ \pm}<g$ & 0.11 \\
$\mathrm{mSP}[\mathrm{t} 1 \mathrm{~b}]$ & $t_{1}<\chi_{2}^{0}<\chi_{1}^{ \pm}<\tau_{1}$ & 0.06 \\
$\mathrm{mSP}[\mathrm{t} 1 \mathrm{c}]$ & $t_{1}<\chi_{2}^{0}<\chi_{1}^{ \pm}<b_{1}$ & 0.02 \\
\hline $\mathrm{mSP}[\mathrm{N} 1 \mathrm{a}]$ & $\chi_{2}^{0}<\chi_{1}^{ \pm}<H^{0}<A^{0}$ & 3.31 \\
$\mathrm{mSP}[\mathrm{N} 1 \mathrm{~b}]$ & $\chi_{2}^{0}<\chi_{1}^{ \pm}<H^{0}<\chi_{3}^{0}$ & 0.02 \\
$\mathrm{mSP}[\mathrm{N} 1 \mathrm{c}]$ & $\chi_{2}^{0}<\chi_{1}^{ \pm}<H^{0}<\tau_{1}$ & 0.02 \\
\hline $\mathrm{mSP}[\mathrm{N} 2 \mathrm{a}]$ & $\chi_{2}^{0}<\chi_{1}^{ \pm}<\chi_{3}^{0}<H^{0}$ & 0.24 \\
$\mathrm{mSP}[\mathrm{N} 2 \mathrm{~b}]$ & $\chi_{2}^{0}<\chi_{1}^{ \pm}<\chi_{3}^{0}<\chi_{4}^{0}$ & 0.20 \\
\hline $\mathrm{mSP}[\mathrm{N} 3]$ & $\chi_{2}^{0}<\chi_{1}^{ \pm}<\tau_{1}<H^{0}$ & 0.39 \\
\hline $\mathrm{mSP}[\mathrm{N} 4]$ & $\chi_{2}^{0}<\chi_{1}^{ \pm}<g<\chi_{3}^{0}$ & 0.26 \\
\hline $\mathrm{mSP}[\mathrm{N} 5]$ & $\chi_{2}^{0}<\chi_{1}^{ \pm}<t_{1}<g$ & 0.02 \\
\hline $\mathrm{mSP}[\mathrm{N} 6]$ & $\chi_{2}^{0}<H^{0}<\chi_{1}^{ \pm}<A^{0}$ & 0.02 \\
\hline $\mathrm{mSP}[\mathrm{H} 1 \mathrm{a}]$ & $H^{0}<A^{0}<\chi^{ \pm}<\chi_{1}^{ \pm}$ & 0.15 \\
$\mathrm{mSP}[\mathrm{H} 1 \mathrm{~b}]$ & $H^{0}<A^{0}<H^{ \pm}<\chi_{2}^{0}$ & 0.06 \\
\hline $\mathrm{mSP}[\mathrm{H} 2]$ & $H^{0}<A^{0}<\chi_{2}^{0}<\chi_{1}^{ \pm}$ & 0.15 \\
\hline $\mathrm{mSP}[\mathrm{H} 3]$ & 0.02 \\
\hline $\mathrm{mSP}[\mathrm{H} 4]$ & $\left.H^{0}\right]$ \\
\hline
\end{tabular}

Table 5. Sparticle mass hierarchies for the mSUGRA parameter space (Model [1]), where $\chi_{1}^{0}$ is the LSP. The high scale parameters lie in the range $m_{0} \in[0.1,10] \mathrm{TeV}, m_{1 / 2} \in[0.1,1.5] \mathrm{TeV}$, $\frac{A_{0}}{m_{0}} \in[-5,5], \tan \beta \in[2,50], \mu>0$, with the constraints $\Omega h^{2}<0.12, m_{h^{0}}>120 \mathrm{GeV}$. Here and in tables 6 to 9 the last column gives the percentage with which the patterns appear in the scans given in table 1. 


\begin{tabular}{|c|c|c|}
\hline Pattern Label & Mass Hierarchy & $\%$ \\
\hline $\mathrm{nuSP}_{2}[\mathrm{C} 1 \mathrm{a}]$ & $\chi_{1}^{ \pm}<\chi_{2}^{0}<\chi_{3}^{0}<\chi_{4}^{0}$ & 35.71 \\
\hline $\mathrm{nuSP}_{2}[\mathrm{C} 1 \mathrm{~b}]$ & $\chi_{1}^{ \pm}<\chi_{2}^{0}<\chi_{3}^{0}<\chi_{2}^{ \pm}$ & 14.57 \\
\hline $\mathrm{nuSP}_{2}[\mathrm{C} 1 \mathrm{c}]$ & $\chi_{1}^{ \pm}<\chi_{2}^{0}<\chi_{3}^{0}<H^{0}$ & 0.188 \\
\hline $\mathrm{nuSP}_{2}[\mathrm{C} 1 \mathrm{~d}]$ & $\chi_{1}^{ \pm}<\chi_{2}^{0}<\chi_{3}^{0}<g$ & 0.010 \\
\hline $\mathrm{nuSP}_{2}[\mathrm{C} 1 \mathrm{e}]$ & $\chi_{1}^{ \pm}<\chi_{2}^{0}<\chi_{3}^{0}<\tau_{1}$ & 0.009 \\
\hline $\mathrm{nuSP}_{2}[\mathrm{C} 1 \mathrm{f}]$ & $\chi_{1}^{ \pm}<\chi_{2}^{0}<\chi_{3}^{0}<t_{1}$ & 0.003 \\
\hline $\mathrm{nuSP}_{2}[\mathrm{C} 1 \mathrm{~g}]$ & $\chi_{1}^{ \pm}<\chi_{2}^{0}<\chi_{3}^{0}<A^{0}$ & 0.001 \\
\hline $\mathrm{nuSP}_{2}[\mathrm{C} 2 \mathrm{a}]$ & $\chi_{1}^{ \pm}<\chi_{2}^{0}<\tau_{1}<\nu_{\tau}$ & 12.25 \\
\hline $\mathrm{nuSP}_{2}[\mathrm{C} 2 \mathrm{~b}]$ & $\chi_{1}^{ \pm}<\chi_{2}^{0}<\tau_{1}<H^{0}$ & 1.571 \\
\hline $\mathrm{nuSP}_{2}[\mathrm{C} 2 \mathrm{c}]$ & $\chi_{1}^{ \pm}<\chi_{2}^{0}<\tau_{1}<t_{1}$ & 0.907 \\
\hline $\mathrm{nuSP}_{2}[\mathrm{C} 2 \mathrm{~d}]$ & $\chi_{1}^{ \pm}<\chi_{2}^{0}<\tau_{1}<\chi_{3}^{0}$ & 0.778 \\
\hline $\mathrm{nuSP}_{2}[\mathrm{C} 2 \mathrm{e}]$ & $\chi_{1}^{ \pm}<\chi_{2}^{0}<\tau_{1}<\mu_{r}$ & 0.289 \\
\hline $\mathrm{nuSP}_{2}[\mathrm{C} 2 \mathrm{f}]$ & $\chi_{1}^{ \pm}<\chi_{2}^{0}<\tau_{1}<g$ & 0.002 \\
\hline $\mathrm{nuSP}_{2}[\mathrm{C} 3 \mathrm{a}]$ & $\chi_{1}^{ \pm}<\chi_{2}^{0}<g<\chi_{3}^{0}$ & 6.237 \\
\hline $\mathrm{nuSP}_{2}[\mathrm{C} 3 \mathrm{~b}]$ & $\chi_{1}^{ \pm}<\chi_{2}^{0}<g<t_{1}$ & 4.820 \\
\hline $\mathrm{nuSP}_{2}[\mathrm{C} 3 \mathrm{c}]$ & $\chi_{1}^{ \pm}<\chi_{2}^{0}<g<H^{0}$ & 0.083 \\
\hline $\mathrm{nuSP}_{2}[\mathrm{C} 3 \mathrm{~d}]$ & $\chi_{1}^{ \pm}<\chi_{2}^{0}<g<H^{ \pm}$ & 0.016 \\
\hline $\mathrm{nuSP}_{2}[\mathrm{C} 4 \mathrm{a}]$ & $\chi_{1}^{ \pm}<\chi_{2}^{0}<H^{0}<A^{0}$ & 2.767 \\
\hline $\mathrm{nuSP}_{2}[\mathrm{C} 4 \mathrm{~b}]$ & $\chi_{1}^{ \pm}<\chi_{2}^{0}<H^{0}<H^{ \pm}$ & 0.074 \\
\hline $\mathrm{nuSP}_{2}[\mathrm{C} 4 \mathrm{c}]$ & $\chi_{1}^{ \pm}<\chi_{2}^{0}<H^{ \pm}<H^{0}$ & 0.025 \\
\hline $\mathrm{nuSP}_{2}[\mathrm{C} 4 \mathrm{~d}]$ & $\chi_{1}^{ \pm}<\chi_{2}^{0}<A^{0}<H^{0}$ & 0.003 \\
\hline $\mathrm{nuSP}_{2}[\mathrm{C} 4 \mathrm{e}]$ & $\chi_{1}^{ \pm}<\chi_{2}^{0}<A^{0}<\tau_{1}$ & 0.001 \\
\hline $\mathrm{nuSP}_{2}[\mathrm{C} 5 \mathrm{a}]$ & $\chi_{1}^{ \pm}<\chi_{2}^{0}<t_{1}<g$ & 6.015 \\
\hline $\mathrm{nuSP}_{2}[\mathrm{C} 5 \mathrm{~b}]$ & $\chi_{1}^{ \pm}<\chi_{2}^{0}<t_{1}<\tau_{1}$ & 2.746 \\
\hline $\mathrm{nuSP}_{2}[\mathrm{C} 5 \mathrm{c}]$ & $\chi_{1}^{ \pm}<\chi_{2}^{0}<t_{1}<\chi_{3}^{0}$ & 1.490 \\
\hline $\mathrm{nuSP}_{2}[\mathrm{C} 5 \mathrm{~d}]$ & $\chi_{1}^{ \pm}<\chi_{2}^{0}<t_{1}<b_{1}$ & 0.222 \\
\hline $\mathrm{nuSP}_{2}[\mathrm{C} 5 \mathrm{e}]$ & $\chi_{1}^{ \pm}<\chi_{2}^{0}<t_{1}<H^{0}$ & 0.039 \\
\hline $\mathrm{nuSP}_{2}[\mathrm{C} 5 \mathrm{f}]$ & $\chi_{1}^{ \pm}<\chi_{2}^{0}<t_{1}<H^{ \pm}$ & 0.008 \\
\hline $\mathrm{nuSP}_{2}[\mathrm{C6a}]$ & $\chi_{1}^{ \pm}<\tau_{1}<\chi_{2}^{0}<\nu_{\tau}$ & 0.274 \\
\hline $\mathrm{nuSP}_{2}[\mathrm{C} 6 \mathrm{~b}]$ & $\chi_{1}^{ \pm}<\tau_{1}<\chi_{2}^{0}<H^{0}$ & 0.008 \\
\hline $\mathrm{nuSP}_{2}[\mathrm{C} 6 \mathrm{c}]$ & $\chi_{1}^{ \pm}<\tau_{1}<\chi_{2}^{0}<\mu_{r}$ & 0.004 \\
\hline $\mathrm{nuSP}_{2}[\mathrm{C} 6 \mathrm{~d}]$ & $\chi_{1}^{ \pm}<\tau_{1}<\chi_{2}^{0}<t_{1}$ & 0.001 \\
\hline $\mathrm{nuSP}_{2}[\mathrm{C} 7 \mathrm{a}]$ & $\chi_{1}^{ \pm}<\tau_{1}<\nu_{\tau}<\nu_{\mu}$ & 0.023 \\
\hline $\mathrm{nuSP}_{2}[\mathrm{C} 7 \mathrm{~b}]$ & $\chi_{1}^{ \pm}<\tau_{1}<\nu_{\tau}<\chi_{2}^{0}$ & 0.004 \\
\hline $\mathrm{nuSP}_{2}[\mathrm{C} 8 \mathrm{a}]$ & $\chi_{1}^{ \pm}<H^{0}<A^{0}<H^{ \pm}$ & 0.010 \\
\hline $\mathrm{nuSP}_{2}[\mathrm{C} 8 \mathrm{~b}]$ & $\chi_{1}^{ \pm}<H^{0}<A^{0}<\chi_{2}^{0}$ & 0.006 \\
\hline $\mathrm{nuSP}_{2}[\mathrm{C} 8 \mathrm{c}]$ & $\chi_{1}^{ \pm}<A^{0}<H^{0}<\chi_{2}^{0}$ & 0.001 \\
\hline $\mathrm{nuSP}_{2}[\mathrm{C9a}]$ & $\chi_{1}^{ \pm}<t_{1}<\chi_{2}^{0}<g$ & 0.003 \\
\hline $\mathrm{nuSP}_{2}[\mathrm{C} 9 \mathrm{~b}]$ & $\chi_{1}^{ \pm}<t_{1}<\chi_{2}^{0}<\tau_{1}$ & 0.003 \\
\hline $\mathrm{nuSP}_{2}[\mathrm{C} 10]$ & $\chi_{1}^{ \pm}<H^{0}<\chi_{2}^{0}<A^{0}$ & 0.001 \\
\hline $\mathrm{nuSP}_{2}[\mathrm{t} 1 \mathrm{a}]$ & $t_{1}<\chi_{2}^{0}<\chi_{1}^{ \pm}<g$ & 0.030 \\
\hline $\mathrm{nuSP}_{2}[\mathrm{t} 1 \mathrm{~b}]$ & $t_{1}<\chi_{2}^{0}<\chi_{1}^{ \pm}<\tau_{1}$ & 0.009 \\
\hline $\operatorname{nuSP}_{2}[\mathrm{t} 1 \mathrm{c}]$ & $t_{1}<\chi_{2}^{0}<\chi_{1}^{ \pm}<b_{1}$ & 0.004 \\
\hline $\mathrm{nuSP}_{2}[\mathrm{t} 2 \mathrm{a}]$ & $t_{1}<\chi_{2}^{0}<\chi_{1}^{ \pm}<g$ & 0.001 \\
\hline $\mathrm{nuSP}_{2}[\mathrm{t} 2 \mathrm{~b}]$ & $t_{1}<\chi_{2}^{0}<\chi_{1}^{ \pm}<\tau_{1}$ & 0.001 \\
\hline
\end{tabular}

\begin{tabular}{|c|c|c|}
\hline Pattern Label & Mass Hierarchy & $\%$ \\
\hline $\operatorname{nuSP}_{2}[\mathrm{H} 1 \mathrm{a}]$ & $H^{0}<A^{0}<H^{ \pm}<\chi_{1}^{ \pm}$ & 0.016 \\
\hline $\mathrm{nuSP}_{2}[\mathrm{H} 1 \mathrm{~b}]$ & $H^{0}<A^{0}<H^{ \pm}<\chi_{2}^{0}$ & 0.003 \\
\hline $\operatorname{nuSP}_{2}[\mathrm{H} 1 \mathrm{c}]$ & $A^{0}<H^{0}<H^{ \pm}<\chi_{2}^{0}$ & 0.001 \\
\hline $\mathrm{nuSP}_{2}[\mathrm{H} 1 \mathrm{~d}]$ & $A^{0}<H^{0}<H^{ \pm}<\chi_{1}^{ \pm}$ & 0.001 \\
\hline $\mathrm{nuSP}_{2}[\mathrm{H} 2 \mathrm{a}]$ & $H^{0}<A^{0}<\chi_{1}^{ \pm}<\chi_{2}^{0}$ & 0.006 \\
\hline $\operatorname{nuSP}_{2}[\mathrm{H} 2 \mathrm{~b}]$ & $H^{0}<A^{0}<\chi_{1}^{ \pm}<H^{ \pm}$ & 0.002 \\
\hline $\mathrm{nuSP}_{2}[\mathrm{H} 2 \mathrm{c}]$ & $A^{0}<H^{0}<\chi_{1}^{ \pm}<\chi_{2}^{0}$ & 0.001 \\
\hline $\mathrm{nuSP}_{2}[\mathrm{H} 3]$ & $H^{0}<A^{0}<\chi_{2}^{0}<\chi_{1}^{ \pm}$ & 0.002 \\
\hline $\mathrm{nuSP}_{2}[\mathrm{H} 4]$ & $H^{0}<\chi_{1}^{ \pm}<A^{0}<\chi_{2}^{0}$ & 0.001 \\
\hline $\mathrm{nuSP}_{2}[\mathrm{N1a}]$ & $\chi_{2}^{0}<\chi_{1}^{ \pm}<g<\chi_{3}^{0}$ & 2.246 \\
\hline $\mathrm{nuSP}_{2}[\mathrm{~N} 1 \mathrm{~b}]$ & $\chi_{2}^{0}<\chi_{1}^{ \pm}<g<t_{1}$ & 1.774 \\
\hline $\mathrm{nuSP}_{2}[\mathrm{~N} 1 \mathrm{c}]$ & $\chi_{2}^{0}<\chi_{1}^{ \pm}<g<H^{0}$ & 0.065 \\
\hline $\mathrm{nuSP}_{2}[\mathrm{~N} 2 \mathrm{a}]$ & $\chi_{2}^{0}<\chi_{1}^{ \pm}<H^{0}<A^{0}$ & 0.976 \\
\hline $\mathrm{nuSP}_{2}[\mathrm{~N} 2 \mathrm{~b}]$ & $\chi_{2}^{0}<\chi_{1}^{ \pm}<H^{0}<\tau_{1}$ & 0.001 \\
\hline $\mathrm{nuSP}_{2}[\mathrm{~N} 2 \mathrm{c}]$ & $\chi_{2}^{0}<\chi_{1}^{ \pm}<A^{0}<H^{0}$ & 0.001 \\
\hline $\mathrm{nuSP}_{2}[\mathrm{~N} 3 \mathrm{a}]$ & $\chi_{2}^{0}<\chi_{1}^{ \pm}<\chi_{3}^{0}<\chi_{4}^{0}$ & 0.448 \\
\hline $\mathrm{nuSP}_{2}[\mathrm{~N} 3 \mathrm{~b}]$ & $\chi_{2}^{0}<\chi_{1}^{ \pm}<\chi_{3}^{0}<\chi_{2}^{ \pm}$ & 0.049 \\
\hline $\mathrm{nuSP}_{2}[\mathrm{~N} 3 \mathrm{c}]$ & $\chi_{2}^{0}<\chi_{1}^{ \pm}<\chi_{3}^{0}<H^{0}$ & 0.017 \\
\hline $\mathrm{nuSP}_{2}[\mathrm{~N} 3 \mathrm{~d}]$ & $\chi_{2}^{0}<\chi_{1}^{ \pm}<\chi_{3}^{0}<g$ & 0.003 \\
\hline $\mathrm{nuSP}_{2}[\mathrm{~N} 3 \mathrm{e}]$ & $\chi_{2}^{0}<\chi_{1}^{ \pm}<\chi_{3}^{0}<t_{1}$ & 0.001 \\
\hline $\mathrm{nuSP}_{2}[\mathrm{~N} 4 \mathrm{a}]$ & $\chi_{2}^{0}<\chi_{1}^{ \pm}<\tau_{1}<H^{0}$ & 0.442 \\
\hline $\mathrm{nuSP}_{2}[\mathrm{~N} 4 \mathrm{~b}]$ & $\chi_{2}^{0}<\chi_{1}^{ \pm}<\tau_{1}<\nu_{\tau}$ & 0.427 \\
\hline $\mathrm{nuSP}_{2}[\mathrm{~N} 4 \mathrm{c}]$ & $\chi_{2}^{0}<\chi_{1}^{ \pm}<\tau_{1}<t_{1}$ & 0.090 \\
\hline $\mathrm{nuSP}_{2}[\mathrm{~N} 4 \mathrm{~d}]$ & $\chi_{2}^{0}<\chi_{1}^{ \pm}<\tau_{1}<\chi_{3}^{0}$ & 0.010 \\
\hline $\mathrm{nuSP}_{2}[\mathrm{~N} 4 \mathrm{e}]$ & $\chi_{2}^{0}<\chi_{1}^{ \pm}<\tau_{1}<\mu_{r}$ & 0.006 \\
\hline $\mathrm{nuSP}_{2}[\mathrm{~N} 5 \mathrm{a}]$ & $\chi_{2}^{0}<\chi_{1}^{ \pm}<t_{1}<g$ & 0.613 \\
\hline $\operatorname{nuSP}_{2}[\mathrm{~N} 5 \mathrm{~b}]$ & $\chi_{2}^{0}<\chi_{1}^{ \pm}<t_{1}<\tau_{1}$ & 0.248 \\
\hline $\mathrm{nuSP}_{2}[\mathrm{~N} 5 \mathrm{c}]$ & $\chi_{2}^{0}<\chi_{1}^{ \pm}<t_{1}<\chi_{3}^{0}$ & 0.067 \\
\hline $\mathrm{nuSP}_{2}[\mathrm{~N} 5 \mathrm{~d}]$ & $\chi_{2}^{0}<\chi_{1}^{ \pm}<t_{1}<b_{1}$ & 0.012 \\
\hline $\mathrm{nuSP}_{2}[\mathrm{~N} 5 \mathrm{e}]$ & $\chi_{2}^{0}<\chi_{1}^{ \pm}<t_{1}<H^{0}$ & 0.008 \\
\hline $\operatorname{nuSP}_{2}[\tau 1 \mathrm{a}]$ & $\tau_{1}<\chi_{2}^{0}<\chi_{1}^{ \pm}<\nu_{\tau}$ & 0.550 \\
\hline $\operatorname{nuSP}_{2}[\tau 1 \mathrm{~b}]$ & $\tau_{1}<\chi_{2}^{0}<\chi_{1}^{ \pm}<H^{0}$ & 0.345 \\
\hline $\operatorname{nuSP}_{2}[\tau 1 \mathrm{c}]$ & $\tau_{1}<\chi_{2}^{0}<\chi_{1}^{ \pm}<\mu_{r}$ & 0.091 \\
\hline $\operatorname{nuSP}_{2}[\tau 1 \mathrm{~d}]$ & $\tau_{1}<\chi_{2}^{0}<\chi_{1}^{ \pm}<t_{1}$ & 0.021 \\
\hline $\mathrm{nuSP}_{2}[\tau 2 \mathrm{a}]$ & $\tau_{1}<\chi_{1}^{ \pm}<\chi_{2}^{0}<\nu_{\tau}$ & 0.098 \\
\hline $\operatorname{nuSP}_{2}[\tau 2 \mathrm{~b}]$ & $\tau_{1}<\chi_{1}^{ \pm}<\chi_{2}^{0}<\mu_{r}$ & 0.014 \\
\hline $\operatorname{nuSP}_{2}[\tau 2 \mathrm{c}]$ & $\tau_{1}<\chi_{1}^{ \pm}<\chi_{2}^{0}<H^{0}$ & 0.006 \\
\hline $\operatorname{nuSP}_{2}[\tau 2 \mathrm{~d}]$ & $\tau_{1}<\chi_{1}^{ \pm}<\chi_{2}^{0}<t_{1}$ & 0.001 \\
\hline $\mathrm{nuSP}_{2}[\tau 3]$ & $\tau_{1}<\chi_{1}^{ \pm}<\nu_{\tau}<\chi_{2}^{0}$ & 0.001 \\
\hline $\mathrm{nuSP}_{2}[\tau 4 \mathrm{a}]$ & $\tau_{1}<\mu_{r}<e_{r}<\chi_{2}^{0}$ & 0.058 \\
\hline $\mathrm{nuSP}_{2}[\tau 4 \mathrm{~b}]$ & $\tau_{1}<\mu_{r}<e_{r}<\nu_{\tau}$ & 0.047 \\
\hline $\mathrm{nuSP}_{2}[\tau 4 \mathrm{c}]$ & $\tau_{1}<\mu_{r}<e_{r}<t_{1}$ & 0.001 \\
\hline $\mathrm{nuSP}_{2}[\tau 5]$ & $\tau_{1}<H^{0}<A^{0}<H^{ \pm}$ & 0.004 \\
\hline $\mathrm{nuSP}_{2}[\tau 6]$ & $\tau_{1}<\chi_{2}^{0}<\mu_{r}<\chi_{1}^{ \pm}$ & 0.001 \\
\hline
\end{tabular}

Table 6. Sparticle mass hierarchies for the nuSUGRA light chargino case (Model [2]). The high scale parameters lie in the range $m_{0} \in[0.1,10] \mathrm{TeV}, M_{1}=M_{3}=m_{1 / 2} \in[0.1,1.5] \mathrm{TeV}, M_{2}=\alpha m_{1 / 2}$, $\alpha \in\left[\frac{1}{2}, 1\right], \frac{A_{0}}{m_{0}} \in[-5,5], \tan \beta \in[2,50], \mu>0$, with the constraints $\Omega h^{2}<0.12, m_{h^{0}}>120 \mathrm{GeV}$. 


\begin{tabular}{|c|c|c|}
\hline Pattern Label & Mass Hierarchy & $\%$ \\
\hline $\operatorname{nuSP}_{3}[\mathrm{C} 1 \mathrm{a}]$ & $\chi_{1}^{ \pm}<\chi_{2}^{0}<\chi_{3}^{0}<\chi_{4}^{0}$ & 63.273 \\
\hline $\mathrm{nuSP}_{3}[\mathrm{C} 1 \mathrm{~b}]$ & $\chi_{1}^{ \pm}<\chi_{2}^{0}<\chi_{3}^{0}<g$ & 10.263 \\
\hline $\operatorname{nuSP}_{3}[\mathrm{C} 1 \mathrm{c}]$ & $\chi_{1}^{ \pm}<\chi_{2}^{0}<\chi_{3}^{0}<H^{0}$ & 4.587 \\
\hline $\operatorname{nuSP}_{3}[\mathrm{C} 1 \mathrm{~d}]$ & $\chi_{1}^{ \pm}<\chi_{2}^{0}<\chi_{3}^{0}<\tau_{1}$ & 4.243 \\
\hline $\mathrm{nuSP}_{3}[\mathrm{C} 1 \mathrm{e}]$ & $\chi_{1}^{ \pm}<\chi_{2}^{0}<\chi_{3}^{0}<t_{1}$ & 4.549 \\
\hline $\mathrm{nuSP}_{3}[\mathrm{C} 1 \mathrm{f}]$ & $\chi_{1}^{ \pm}<\chi_{2}^{0}<\chi_{3}^{0}<\chi_{2}^{ \pm}$ & 0.482 \\
\hline $\operatorname{nuSP}_{3}[\mathrm{C} 2 \mathrm{a}]$ & $\chi_{1}^{ \pm}<\chi_{2}^{0}<\tau_{1}<\mu_{r}$ & 0.854 \\
\hline $\mathrm{nuSP}_{3}[\mathrm{C} 2 \mathrm{~b}]$ & $\chi_{1}^{ \pm}<\chi_{2}^{0}<\tau_{1}<\chi_{3}^{0}$ & 0.647 \\
\hline $\mathrm{nuSP}_{3}[\mathrm{C} 2 \mathrm{c}]$ & $\chi_{1}^{ \pm}<\chi_{2}^{0}<\tau_{1}<t_{1}$ & 0.372 \\
\hline $\mathrm{nuSP}_{3}[\mathrm{C} 2 \mathrm{~d}]$ & $\chi_{1}^{ \pm}<\chi_{2}^{0}<\tau_{1}<H^{0}$ & 0.138 \\
\hline $\mathrm{nuSP}_{3}[\mathrm{C} 3 \mathrm{a}]$ & $\chi_{1}^{ \pm}<\chi_{2}^{0}<t_{1}<\chi_{3}^{0}$ & 0.840 \\
\hline $\mathrm{nuSP}_{3}[\mathrm{C} 3 \mathrm{~b}]$ & $\chi_{1}^{ \pm}<\chi_{2}^{0}<t_{1}<\tau_{1}$ & 0.716 \\
\hline $\operatorname{nuSP}_{3}[\mathrm{C} 3 \mathrm{c}]$ & $\chi_{1}^{ \pm}<\chi_{2}^{0}<t_{1}<H^{0}$ & 0.055 \\
\hline $\mathrm{nuSP}_{3}[\mathrm{C} 3 \mathrm{~d}]$ & $\chi_{1}^{ \pm}<\chi_{2}^{0}<t_{1}<g$ & 0.028 \\
\hline $\mathrm{nuSP}_{3}[\mathrm{C} 4]$ & $\chi_{1}^{ \pm}<\chi_{2}^{0}<H^{0}<A^{0}$ & 0.882 \\
\hline $\mathrm{nuSP}_{3}[\mathrm{C} 5]$ & $\chi_{1}^{ \pm}<\chi_{2}^{0}<g<\chi_{3}^{0}$ & 0.523 \\
\hline $\mathrm{nuSP}_{3}[\mathrm{C} 6 \mathrm{a}]$ & $\chi_{1}^{ \pm}<\tau_{1}<\chi_{2}^{0}<\mu_{r}$ & 0.096 \\
\hline $\mathrm{nuSP}_{3}[\mathrm{C} 6 \mathrm{~b}]$ & $\chi_{1}^{ \pm}<\tau_{1}<\chi_{2}^{0}<\chi_{3}^{0}$ & 0.096 \\
\hline $\mathrm{nuSP}_{3}[\mathrm{C} 6 \mathrm{c}]$ & $\chi_{1}^{ \pm}<\tau_{1}<\chi_{2}^{0}<H^{0}$ & 0.014 \\
\hline $\operatorname{nuSP}_{3}[\mathrm{C} 6 \mathrm{~d}]$ & $\chi_{1}^{ \pm}<\tau_{1}<\chi_{2}^{0}<t_{1}$ & 0.014 \\
\hline $\operatorname{nuSP}_{3}[\mathrm{C} 7]$ & $\chi_{1}^{ \pm}<g<\chi_{2}^{0}<\chi_{3}^{0}$ & 0.055 \\
\hline $\mathrm{nuSP}_{3}[\mathrm{C} 8]$ & $\chi_{1}^{ \pm}<t_{1}<\chi_{2}^{0}<\chi_{3}^{0}$ & 0.041 \\
\hline $\mathrm{nuSP}_{3}[\mathrm{~N} 1 \mathrm{a}]$ & $\chi_{2}^{0}<\chi_{1}^{ \pm}<H^{0}<A^{0}$ & 0.978 \\
\hline $\mathrm{nuSP}_{3}[\mathrm{~N} 1 \mathrm{~b}]$ & $\chi_{2}^{0}<\chi_{1}^{ \pm}<A^{0}<H^{0}$ & 0.014 \\
\hline $\mathrm{nuSP}_{3}[\mathrm{~N} 2]$ & $\chi_{2}^{0}<\chi_{1}^{ \pm}<g<\chi_{3}^{0}$ & 0.193 \\
\hline $\operatorname{nuSP}_{3}[\mathrm{~N} 3 \mathrm{a}]$ & $\chi_{2}^{0}<\chi_{1}^{ \pm}<\tau_{1}<H^{0}$ & 0.083 \\
\hline $\mathrm{nuSP}_{3}[\mathrm{~N} 3 \mathrm{~b}]$ & $\chi_{2}^{0}<\chi_{1}^{ \pm}<\tau_{1}<\chi_{3}^{0}$ & 0.028 \\
\hline $\mathrm{nuSP}_{3}[\mathrm{~N} 4]$ & $\chi_{2}^{0}<\chi_{1}^{ \pm}<t_{1}<H^{0}$ & 0.041 \\
\hline $\mathrm{nuSP}_{3}[\mathrm{~N} 5]$ & $\chi_{2}^{0}<\chi_{1}^{ \pm}<\chi_{3}^{0}<H^{0}$ & 0.014 \\
\hline $\operatorname{nuSP}_{3}[\mathrm{~g} 1 \mathrm{a}]$ & $g<\chi_{2}^{0}<\chi_{1}^{ \pm}<\chi_{3}^{0}$ & 3.940 \\
\hline $\mathrm{nuSP}_{3}[\mathrm{~g} 1 \mathrm{~b}]$ & $g<\chi_{2}^{0}<\chi_{1}^{ \pm}<t_{1}$ & 3.031 \\
\hline $\mathrm{nuSP}_{3}[\mathrm{~g} 1 \mathrm{c}]$ & $g<\chi_{2}^{0}<\chi_{1}^{ \pm}<H^{0}$ & 0.138 \\
\hline $\operatorname{nuSP}_{3}[\mathrm{~g} 1 \mathrm{~d}]$ & $g<\chi_{2}^{0}<\chi_{1}^{ \pm}<A^{0}$ & 0.014 \\
\hline $\mathrm{nuSP}_{3}[\mathrm{~g} 1 \mathrm{e}]$ & $g<\chi_{2}^{0}<\chi_{1}^{ \pm}<H^{ \pm}$ & 0.014 \\
\hline $\operatorname{nuSP}_{3}[\mathrm{~g} 2 \mathrm{a}]$ & $g<\chi_{1}^{ \pm}<\chi_{2}^{0}<\chi_{3}^{0}$ & 1.529 \\
\hline $\mathrm{nuSP}_{3}[\mathrm{~g} 2 \mathrm{~b}]$ & $g<\chi_{1}^{ \pm}<\chi_{2}^{0}<t_{1}$ & 0.028 \\
\hline $\mathrm{nuSP}_{3}[\mathrm{~g} 3]$ & $g<t_{1}<\chi_{2}^{0}<\chi_{1}^{ \pm}$ & 0.413 \\
\hline $\mathrm{nuSP}_{3}[\mathrm{~g} 4]$ & $g<t_{1}<\chi_{1}^{ \pm}<\chi_{2}^{0}$ & 0.124 \\
\hline $\mathrm{nuSP}_{3}[\mathrm{~g} 5]$ & $g<t_{1}<H^{0}<A^{0}$ & 0.014 \\
\hline $\mathrm{nuSP}_{3}[\mathrm{~g} 6]$ & $g<H^{0}<A^{0}<H^{ \pm}$ & 0.069 \\
\hline $\mathrm{nuSP}_{3}[\mathrm{H} 1]$ & $H^{0}<A^{0}<\chi_{2}^{0}<\chi_{1}^{ \pm}$ & 0.096 \\
\hline $\mathrm{nuSP}_{3}[\mathrm{H} 2]$ & $H^{0}<A^{0}<\chi_{2}^{0}<H^{ \pm}$ & 0.014 \\
\hline $\operatorname{nuSP}_{3}[\mathrm{H} 3 \mathrm{a}]$ & $H^{0}<A^{0}<H^{ \pm}<\chi_{1}^{ \pm}$ & 0.069 \\
\hline $\mathrm{nuSP}_{3}[\mathrm{H} 3 \mathrm{~b}]$ & $H^{0}<A^{0}<H^{ \pm}<\chi_{2}^{0}$ & 0.055 \\
\hline $\mathrm{nuSP}_{3}[\mathrm{H} 3 \mathrm{c}]$ & $H^{0}<A^{0}<H^{ \pm}<t_{1}$ & 0.014 \\
\hline $\mathrm{nuSP}_{3}[\mathrm{~A} 1]$ & $A^{0}<H^{0}<\chi_{1}^{ \pm}<\chi_{2}^{0}$ & 0.014 \\
\hline
\end{tabular}

\begin{tabular}{|c|c|c|}
\hline Pattern Label & Mass Hierarchy & $\%$ \\
\hline $\operatorname{nuSP}_{3}[\mathrm{t} 1 \mathrm{a}]$ & $t_{1}<\chi_{2}^{0}<\chi_{1}^{ \pm}<g$ & 0.992 \\
\hline $\operatorname{nuSP}_{3}[\mathrm{t} 1 \mathrm{~b}]$ & $t_{1}<\chi_{2}^{0}<\chi_{1}^{ \pm}<\tau_{!}$ & 0.427 \\
\hline $\operatorname{nuSP}_{3}[\mathrm{t} 1 \mathrm{c}]$ & $t_{1}<\chi_{2}^{0}<\chi_{1}^{ \pm}<H^{0}$ & 0.041 \\
\hline $\mathrm{nuSP}_{3}[\mathrm{t} 1 \mathrm{~d}]$ & $t_{1}<\chi_{2}^{0}<\chi_{1}^{ \pm}<\chi_{3}^{0}$ & 0.014 \\
\hline $\mathrm{nuSP}_{3}[\mathrm{t} 2 \mathrm{a}]$ & $t_{1}<\chi_{1}^{ \pm}<\chi_{2}^{0}<\chi_{3}^{0}$ & 0.730 \\
\hline $\mathrm{nuSP}_{3}[\mathrm{t} 2 \mathrm{~b}]$ & $t_{1}<\chi_{1}^{ \pm}<\chi_{2}^{0}<\tau_{1}$ & 0.069 \\
\hline $\mathrm{nuSP}_{3}[\mathrm{t} 2 \mathrm{c}]$ & $t_{1}<\chi_{1}^{ \pm}<\chi_{2}^{0}<g$ & 0.014 \\
\hline $\mathrm{nuSP}_{3}[\mathrm{t} 2 \mathrm{~d}]$ & $t_{1}<\chi_{1}^{ \pm}<\chi_{2}^{0}<H^{0}$ & 0.014 \\
\hline $\mathrm{nuSP}_{3}[\mathrm{t} 3]$ & $t_{1}<\tau_{1}<\mu_{r}<e_{r}$ & 0.386 \\
\hline $\mathrm{nuSP}_{3}[\mathrm{t} 4]$ & $t_{1}<g<\chi_{2}^{0}<\chi_{1}^{ \pm}$ & 0.344 \\
\hline $\mathrm{nuSP}_{3}[\mathrm{t} 5]$ & $t_{1}<\tau_{1}<\chi_{2}^{0}<\chi_{1}^{ \pm}$ & 0.303 \\
\hline $\operatorname{nuSP}_{3}[\mathrm{t} 6 \mathrm{a}]$ & $t_{1}<\tau_{1}<\chi_{1}^{ \pm}<\chi_{2}^{0}$ & 0.165 \\
\hline $\operatorname{nuSP}_{3}[\mathrm{t} 6 \mathrm{~b}]$ & $t_{1}<\tau_{1}<\chi_{1}^{ \pm}<\mu_{r}$ & 0.014 \\
\hline $\mathrm{nuSP}_{3}[\mathrm{t} 7]$ & $t_{1}<g<\chi_{1}^{ \pm}<\chi_{3}^{0}$ & 0.152 \\
\hline $\mathrm{nuSP}_{3}[\mathrm{t} 8]$ & $t_{1}<\tau_{1}<H^{0}<A^{0}$ & 0.041 \\
\hline $\operatorname{nuSP}_{3}[\mathrm{t} 9]$ & $t_{1}<\chi_{1}^{ \pm}<\tau_{1}<\chi_{2}^{0}$ & 0.028 \\
\hline $\mathrm{nuSP}_{3}[\mathrm{t} 10]$ & $t_{1}<g<H^{0}<A^{0}$ & 0.014 \\
\hline $\mathrm{nuSP}_{3}[\mathrm{t} 11 \mathrm{a}]$ & $t_{1}<H^{0}<A^{0}<H^{ \pm}$ & 0.014 \\
\hline $\mathrm{nuSP}_{3}[\mathrm{t} 11 \mathrm{~b}]$ & $t_{1}<H^{0}<A^{0}<\chi_{2}^{0}$ & 0.014 \\
\hline $\mathrm{nuSP}_{3}[\mathrm{t} 12]$ & $t_{1}<\chi_{1}^{ \pm}<g<\chi_{2}^{0}$ & 0.014 \\
\hline $\mathrm{nuSP}_{3}[\tau 1 \mathrm{a}]$ & $\tau_{1}<\mu_{r}<e_{r}<\chi_{1}^{ \pm}$ & 1.681 \\
\hline $\mathrm{nuSP}_{3}[\tau 1 \mathrm{~b}]$ & $\tau_{1}<\mu_{r}<e_{r}<t_{1}$ & 0.234 \\
\hline $\operatorname{nuSP}_{3}[\tau 1 \mathrm{c}]$ & $\tau_{1}<\mu_{r}<e_{r}<\nu_{\tau}$ & 0.207 \\
\hline $\operatorname{nuSP}_{3}[\tau 1 \mathrm{~d}]$ & $\tau_{1}<\mu_{r}<e_{r}<\chi_{2}^{0}$ & 0.207 \\
\hline $\mathrm{nuSP}_{3}[\tau 2 \mathrm{a}]$ & $\tau_{1}<\chi_{1}^{ \pm}<\chi_{2}^{0}<\chi_{3}^{0}$ & 0.951 \\
\hline $\mathrm{nuSP}_{3}[\tau 2 \mathrm{~b}]$ & $\tau_{1}<\chi_{1}^{ \pm}<\chi_{2}^{0}<\mu_{r}$ & 0.152 \\
\hline $\operatorname{nuSP}_{3}[\tau 2 \mathrm{c}]$ & $\tau_{1}<\chi_{1}^{ \pm}<\chi_{2}^{0}<t_{1}$ & 0.069 \\
\hline $\mathrm{nuSP}_{3}[\tau 2 \mathrm{~d}]$ & $\tau_{1}<\chi_{1}^{ \pm}<\chi_{2}^{0}<H^{0}$ & 0.028 \\
\hline $\mathrm{nuSP}_{3}[\tau 3 \mathrm{a}]$ & $\tau_{1}<\chi_{2}^{0}<\chi_{1}^{ \pm}<H^{0}$ & 0.661 \\
\hline $\mathrm{nuSP}_{3}[\tau 3 \mathrm{~b}]$ & $\tau_{1}<\chi_{2}^{0}<\chi_{1}^{ \pm}<\chi_{3}^{0}$ & 0.096 \\
\hline $\operatorname{nuSP}_{3}[\tau 3 c]$ & $\tau_{1}<\chi_{2}^{0}<\chi_{1}^{ \pm}<\mu_{r}$ & 0.069 \\
\hline $\mathrm{nuSP}_{3}[\tau 3 \mathrm{~d}]$ & $\tau_{1}<\chi_{2}^{0}<\chi_{1}^{ \pm}<\nu_{\tau}$ & 0.028 \\
\hline $\operatorname{nuSP}_{3}[\tau 4 \mathrm{a}]$ & $\tau_{1}<H^{0}<A^{0}<H^{ \pm}$ & 0.069 \\
\hline $\mathrm{nuSP}_{3}[\tau 4 \mathrm{~b}]$ & $\tau_{1}<H^{0}<A^{0}<\chi_{2}^{0}$ & 0.014 \\
\hline $\mathrm{nuSP}_{3}[\tau 5]$ & $\tau_{1}<t_{1}<\chi_{2}^{0}<\chi_{1}^{ \pm}$ & 0.124 \\
\hline $\mathrm{nuSP}_{3}[\tau 6]$ & $\tau_{1}<t_{1}<\mu_{r}<e_{r}$ & 0.110 \\
\hline $\operatorname{nuSP}_{3}[\tau 7]$ & $\tau_{1}<t_{1}<\chi_{1}^{ \pm}<\chi_{2}^{0}$ & 0.083 \\
\hline $\mathrm{nuSP}_{3}[\tau 8]$ & $\tau_{1}<t_{1}<H^{0}<A^{0}$ & 0.028 \\
\hline $\mathrm{nuSP}_{3}[\tau 9]$ & $\tau_{1}<\chi_{1}^{ \pm}<\mu_{r}<e_{r}$ & 0.028 \\
\hline
\end{tabular}

Table 7. Sparticle mass hierarchies for the nuSUGRA light gluino case (Model [3]). The high scale parameters lie in the range $m_{0} \in[0.1,10] \mathrm{TeV}, M_{1}=M_{2}=m_{1 / 2} \in[0.1,1.5] \mathrm{TeV}, M_{3}=\alpha m_{1 / 2}$, $\alpha \in\left[\frac{1}{6}, 1\right], \frac{A_{0}}{m_{0}} \in[-5,5], \tan \beta \in[2,50], \mu>0$, with the constraints $\Omega h^{2}<0.12, m_{h^{0}}>120 \mathrm{GeV}$. 


\begin{tabular}{|c|c|c|}
\hline Pattern Label & Mass Hierarchy & $\%$ \\
\hline $\mathrm{nuSP}_{4}[\mathrm{C} 1 \mathrm{a}]$ & $\chi_{1}^{ \pm}<\chi_{2}^{0}<\chi_{3}^{0}<\chi_{4}^{0}$ & 55.065 \\
\hline $\mathrm{nuSP}_{4}[\mathrm{C} 1 \mathrm{~b}]$ & $\chi_{1}^{ \pm}<\chi_{2}^{0}<\chi_{3}^{0}<\tau_{1}$ & 12.727 \\
\hline $\mathrm{nuSP}_{4}[\mathrm{C} 1 \mathrm{c}]$ & $\chi_{1}^{ \pm}<\chi_{2}^{0}<\chi_{3}^{0}<A^{0}$ & 6.205 \\
\hline $\mathrm{nuSP}_{4}[\mathrm{C} 1 \mathrm{~d}]$ & $\chi_{1}^{ \pm}<\chi_{2}^{0}<\chi_{3}^{0}<H^{0}$ & 2.870 \\
\hline $\mathrm{nuSP}_{4}[\mathrm{C} 1 \mathrm{e}]$ & $\chi_{1}^{ \pm}<\chi_{2}^{0}<\chi_{3}^{0}<t_{1}$ & 0.886 \\
\hline $\mathrm{nuSP}_{4}[\mathrm{C} 1 \mathrm{f}]$ & $\chi_{1}^{ \pm}<\chi_{2}^{0}<\chi_{3}^{0}<\chi_{2}^{ \pm}$ & 0.570 \\
\hline $\operatorname{nuSP}_{4}[\mathrm{C} 1 \mathrm{~g}]$ & $\chi_{1}^{ \pm}<\chi_{2}^{0}<\chi_{3}^{0}<H^{ \pm}$ & 0.507 \\
\hline $\mathrm{nuSP}_{4}[\mathrm{C} 2 \mathrm{a}]$ & $\chi_{1}^{ \pm}<\chi_{2}^{0}<A^{0}<H^{0}$ & 1.224 \\
\hline $\mathrm{nuSP}_{4}[\mathrm{C} 2 \mathrm{~b}]$ & $\chi_{1}^{ \pm}<\chi_{2}^{0}<H^{0}<A^{0}$ & 0.549 \\
\hline $\mathrm{nuSP}_{4}[\mathrm{C} 3 \mathrm{a}]$ & $\chi_{1}^{ \pm}<\chi_{2}^{0}<\tau_{1}<\chi_{3}^{0}$ & 1.203 \\
\hline $\mathrm{nuSP}_{4}[\mathrm{C} 3 \mathrm{~b}]$ & $\chi_{1}^{ \pm}<\chi_{2}^{0}<\tau_{1}<H^{0}$ & 0.042 \\
\hline $\mathrm{nuSP}_{4}[\mathrm{C} 3 \mathrm{c}]$ & $\chi_{1}^{ \pm}<\chi_{2}^{0}<\tau_{1}<A^{0}$ & 0.021 \\
\hline $\mathrm{nuSP}_{4}[\mathrm{C} 4]$ & $\chi_{1}^{ \pm}<\chi_{2}^{0}<t_{1}<\chi_{3}^{0}$ & 0.169 \\
\hline $\mathrm{nuSP}_{4}[\mathrm{C} 5]$ & $\chi_{1}^{ \pm}<A^{0}<H^{0}<\chi_{2}^{0}$ & 0.084 \\
\hline $\mathrm{nuSP}_{4}[\mathrm{C} 6]$ & $\chi_{1}^{ \pm}<\chi_{2}^{0}<g<\chi_{3}^{0}$ & 0.063 \\
\hline $\mathrm{nuSP}_{4}[\mathrm{C} 7]$ & $\chi_{1}^{ \pm}<\tau_{1}<\chi_{2}^{0}<\chi_{3}^{0}<$ & 0.021 \\
\hline $\mathrm{nuSP}_{4}[\mathrm{C} 8]$ & $\chi_{1}^{ \pm}<\chi_{2}^{0}<H^{ \pm}<A^{0}$ & 0.021 \\
\hline $\operatorname{nuSP}_{4}[\tau 1 \mathrm{a}]$ & $\tau_{1}<\chi_{2}^{0}<\chi_{1}^{ \pm}<H^{0}$ & 2.617 \\
\hline $\operatorname{nuSP}_{4}[\tau 1 \mathrm{~b}]$ & $\tau_{1}<\chi_{2}^{0}<\chi_{1}^{ \pm}<\mu_{r}$ & 0.971 \\
\hline $\mathrm{nuSP}_{4}[\tau 1 \mathrm{c}]$ & $\tau_{1}<\chi_{2}^{0}<\chi_{1}^{ \pm}<\nu_{\tau}$ & 0.802 \\
\hline $\operatorname{nuSP}_{4}[\tau 1 \mathrm{~d}]$ & $\tau_{1}<\chi_{2}^{0}<\chi_{1}^{ \pm}<t_{1}$ & 0.106 \\
\hline $\mathrm{nuSP}_{4}[\tau 1 \mathrm{e}]$ & $\tau_{1}<\chi_{2}^{0}<\chi_{1}^{ \pm}<\chi_{3}^{0}$ & 0.021 \\
\hline $\mathrm{nuSP}_{4}[\tau 2 \mathrm{a}]$ & $\tau_{1}<\mu_{r}<e_{r}<\chi_{2}^{0}$ & 2.849 \\
\hline $\mathrm{nuSP}_{4}[\tau 2 \mathrm{~b}]$ & $\tau_{1}<\mu_{r}<e_{r}<\nu_{\tau}$ & 2.153 \\
\hline $\mathrm{nuSP}_{4}[\tau 2 \mathrm{c}]$ & $\tau_{1}<\mu_{r}<e_{r}<\chi_{1}^{ \pm}$ & 1.224 \\
\hline $\mathrm{nuSP}_{4}[\tau 2 \mathrm{~d}]$ & $\tau_{1}<\mu_{r}<e_{r}<H^{0}$ & 0.021 \\
\hline $\operatorname{nuSP}_{4}[\tau 3 \mathrm{a}]$ & $\tau_{1}<\chi_{1}^{ \pm}<\chi_{2}^{0}<\chi_{3}^{0}$ & 1.625 \\
\hline $\mathrm{nuSP}_{4}[\tau 3 \mathrm{~b}]$ & $\tau_{1}<\chi_{1}^{ \pm}<\chi_{2}^{0}<\mu_{r}$ & 0.295 \\
\hline $\mathrm{nuSP}_{4}[\tau 3 \mathrm{c}]$ & $\tau_{1}<\chi_{1}^{ \pm}<\chi_{2}^{0}<H^{0}$ & 0.084 \\
\hline $\mathrm{nuSP}_{4}[\tau 4 \mathrm{a}]$ & $\tau_{1}<H^{0}<A^{0}<H^{ \pm}$ & 0.148 \\
\hline $\mathrm{nuSP}_{4}[\tau 4 \mathrm{~b}]$ & $\tau_{1}<H^{0}<A^{0}<\chi_{2}^{0}$ & 0.063 \\
\hline $\mathrm{nuSP}_{4}[\tau 5]$ & $\tau_{1}<t_{1}<\chi_{2}^{0}<\chi_{1}^{ \pm}$ & 0.021 \\
\hline $\mathrm{nuSP}_{4}[\tau 6]$ & $\tau_{1}<\mu_{r}<\chi_{2}^{0}<e_{r}$ & 0.021 \\
\hline
\end{tabular}

\begin{tabular}{|c|c|c|}
\hline Pattern Label & Mass Hierarchy & $\%$ \\
\hline $\mathrm{nuSP}_{4}[\mathrm{~N} 1 \mathrm{a}]$ & $\chi_{2}^{0}<\chi_{1}^{ \pm}<H^{0}<A^{0}$ & 2.216 \\
\hline $\mathrm{nuSP}_{4}[\mathrm{~N} 1 \mathrm{~b}]$ & $\chi_{2}^{0}<\chi_{1}^{ \pm}<A^{0}<H^{0}$ & 0.443 \\
\hline $\mathrm{nuSP}_{4}[\mathrm{~N} 2 \mathrm{a}]$ & $\chi_{2}^{0}<\chi_{1}^{ \pm}<\tau_{1}<H^{0}$ & 0.401 \\
\hline $\mathrm{nuSP}_{4}[\mathrm{~N} 2 \mathrm{~b}]$ & $\chi_{2}^{0}<\chi_{1}^{ \pm}<\tau_{1}<A^{0}$ & 0.042 \\
\hline $\mathrm{nuSP}_{4}[\mathrm{~N} 2 \mathrm{c}]$ & $\chi_{2}^{0}<\chi_{1}^{ \pm}<\tau_{1}<\chi_{3}^{0}$ & 0.021 \\
\hline $\mathrm{nuSP}_{4}[\mathrm{~N} 3]$ & $\chi_{2}^{0}<\chi_{1}^{ \pm}<g<\chi_{3}^{0}$ & 0.106 \\
\hline $\mathrm{nuSP}_{4}[\mathrm{~N} 4 \mathrm{a}]$ & $\chi_{2}^{0}<\chi_{1}^{ \pm}<\chi_{3}^{0}<\chi_{4}^{0}$ & 0.063 \\
\hline $\mathrm{nuSP}_{4}[\mathrm{~N} 4 \mathrm{~b}]$ & $\chi_{2}^{0}<\chi_{1}^{ \pm}<\chi_{3}^{0}<\tau_{1}$ & 0.042 \\
\hline $\mathrm{nuSP}_{4}[\mathrm{~N} 4 \mathrm{c}]$ & $\chi_{2}^{0}<\chi_{1}^{ \pm}<\chi_{3}^{0}<A^{0}$ & 0.042 \\
\hline $\mathrm{nuSP}_{4}[\mathrm{~N} 4 \mathrm{~d}]$ & $\chi_{2}^{0}<\chi_{1}^{ \pm}<\chi_{3}^{0}<H^{ \pm}$ & 0.021 \\
\hline $\mathrm{nuSP}_{4}[\mathrm{~N} 5]$ & $\chi_{2}^{0}<\chi_{1}^{ \pm}<t_{1}<g$ & 0.021 \\
\hline $\mathrm{nuSP}_{4}[\mathrm{t} 1 \mathrm{a}]$ & $t_{1}<\chi_{2}^{0}<\chi_{1}^{ \pm}<g$ & 0.359 \\
\hline $\mathrm{nuSP}_{4}[\mathrm{t} 1 \mathrm{~b}]$ & $t_{1}<\chi_{2}^{0}<\chi_{1}^{ \pm}<\chi_{3}^{0}$ & 0.317 \\
\hline $\mathrm{nuSP}_{4}[\mathrm{t} 1 \mathrm{c}]$ & $t_{1}<\chi_{2}^{0}<\chi_{1}^{ \pm}<\tau_{1}$ & 0.127 \\
\hline $\mathrm{nuSP}_{4}[\mathrm{t} 1 \mathrm{~d}]$ & $t_{1}<\chi_{2}^{0}<\chi_{1}^{ \pm}<H^{0}$ & 0.042 \\
\hline $\mathrm{nuSP}_{4}[\mathrm{t} 1 \mathrm{e}]$ & $t_{1}<\chi_{2}^{0}<\chi_{1}^{ \pm}<b_{1}$ & 0.021 \\
\hline $\mathrm{nuSP}_{4}[\mathrm{t} 2 \mathrm{a}]$ & $t_{1}<\chi_{1}^{ \pm}<\chi_{2}^{0}<\chi_{3}^{0}$ & 0.106 \\
\hline $\mathrm{nuSP}_{4}[\mathrm{t} 2 \mathrm{~b}]$ & $t_{1}<\chi_{1}^{ \pm}<\chi_{2}^{0}<H^{0}$ & 0.021 \\
\hline $\mathrm{nuSP}_{4}[\mathrm{t} 3 \mathrm{a}]$ & $t_{1}<H^{0}<A^{0}<H^{ \pm}$ & 0.021 \\
\hline $\mathrm{nuSP}_{4}[\mathrm{t} 3 \mathrm{a}]$ & $t_{1}<H^{0}<A^{0}<\chi_{1}^{ \pm}$ & 0.021 \\
\hline $\mathrm{nuSP}_{4}[\mathrm{H} 1 \mathrm{a}]$ & $H^{0}<A^{0}<H^{ \pm}<\chi_{1}^{ \pm}$ & 0.127 \\
\hline $\mathrm{nuSP}_{4}[\mathrm{H} 1 \mathrm{~b}]$ & $H^{0}<A^{0}<H^{ \pm}<\chi_{2}^{0}$ & 0.021 \\
\hline $\mathrm{nuSP}_{4}[\mathrm{H} 2 \mathrm{a}]$ & $H^{0}<A^{0}<\chi_{2}^{0}<\chi_{1}^{ \pm}$ & 0.084 \\
\hline $\mathrm{nuSP}_{4}[\mathrm{H} 2 \mathrm{~b}]$ & $A^{0}<H^{0}<\chi_{2}^{0}<\chi_{1}^{ \pm}$ & 0.021 \\
\hline $\mathrm{nuSP}_{4}[\mathrm{H} 3]$ & $A^{0}<H^{0}<\chi_{1}^{ \pm}<H^{ \pm}$ & 0.021 \\
\hline $\mathrm{nuSP}_{4}[\mathrm{H} 4]$ & $H^{0}<\chi_{2}^{0}<\chi_{1}^{ \pm}<H^{ \pm}$ & 0.021 \\
\hline
\end{tabular}

Table 8. Sparticle mass hierarchies for the nuSUGRA nonuniversal Higgs case (Model [4]). The high scale parameters lie in the range $m_{0} \in[0.1,10] \mathrm{TeV}, m_{1 / 2} \in[0.1,1.5] \mathrm{TeV}, \frac{A_{0}}{m_{0}} \in[-5,5]$, $\tan \beta \in[2,50], \mu>0$, with the constraints $\Omega h^{2}<0.12, m_{h^{0}}>120 \mathrm{GeV}$. However, the Higgs masses at the GUT scale are nonuniversal, $m_{H_{i}}\left(\mathrm{M}_{G}\right)=m_{0}\left(1+\delta_{i}\right), i=1,2$ where $\delta_{i} \in[-0.9,1]$. 


\begin{tabular}{|l|l|c|}
\hline Pattern Label & \multicolumn{1}{|c|}{ Mass Hierarchy } & $\%$ \\
\hline $\operatorname{nuSP}_{5}[\mathrm{C} 1 \mathrm{a}]$ & $\chi_{1}^{ \pm}<\chi_{2}^{0}<\chi_{3}^{0}<\tau_{1}$ & 39.4 \\
$\operatorname{nuSP}_{5}[\mathrm{C} 1 \mathrm{~b}]$ & $\chi_{1}^{ \pm}<\chi_{2}^{0}<\chi_{3}^{0}<\chi_{4}^{0}$ & 12.5 \\
$\operatorname{nuSP}_{5}[\mathrm{C} 1 \mathrm{c}]$ & $\chi_{1}^{ \pm}<\chi_{2}^{0}<\chi_{3}^{0}<t_{1}$ & 1.39 \\
$\operatorname{nuSP}_{5}[\mathrm{C} 1 \mathrm{~d}]$ & $\chi_{1}^{ \pm}<\chi_{2}^{0}<\chi_{3}^{0}<H^{0}$ & 0.37 \\
\hline $\operatorname{nuSP}_{5}[\mathrm{C} 2 \mathrm{a}]$ & $\chi_{1}^{ \pm}<\chi_{2}^{0}<\tau_{1}<\chi_{3}^{0}$ & 17.95 \\
$\operatorname{nuSP}_{5}[\mathrm{C} 2 \mathrm{~b}]$ & $\chi_{1}^{ \pm}<\chi_{2}^{0}<\tau_{1}<H^{0}$ & 0.04 \\
$\operatorname{nuSP}_{5}[\mathrm{C} 2 \mathrm{c}]$ & $\chi_{1}^{ \pm}<\chi_{2}^{0}<\tau_{1}<t_{1}$ & 0.04 \\
\hline $\operatorname{nuSP}_{5}[\mathrm{C} 3]$ & $\chi_{1}^{ \pm}<\tau_{1}<\chi_{2}^{0}<\chi_{3}^{0}$ & 0.49 \\
\hline $\operatorname{nuSP}_{5}[\mathrm{C} 4]$ & $\chi_{1}^{ \pm}<\chi_{2}^{0}<H^{0}<A^{0}$ & 0.22 \\
\hline $\operatorname{nuSP}_{5}[\mathrm{C} 5 \mathrm{a}]$ & $\chi_{1}^{ \pm}<\chi_{2}^{0}<t_{1}<\chi_{3}^{0}$ & 0.07 \\
$\operatorname{nuSP}_{5}[\mathrm{C} 5 \mathrm{~b}]$ & $\chi_{1}^{ \pm}<\chi_{2}^{0}<t_{1}<\tau_{1}$ & 0.04 \\
\hline $\operatorname{nuSP}_{5}[\mathrm{e} 1]$ & $e_{r}<\mu_{r}<\nu_{e}<\nu_{\mu}$ & 0.19 \\
\hline $\operatorname{nuSP}_{5}[\mathrm{H} 1]$ & $H^{0}<A^{0}<\chi_{2}^{0}<\chi_{1}^{ \pm}$ & 0.07 \\
\hline $\operatorname{nuSP}_{5}[\mu 1]$ & $\mu_{r}<e_{r}<\nu_{\mu}<\nu_{e}$ & 3.37 \\
\hline $\operatorname{nuSP}_{5}[\mu 2 \mathrm{a}]$ & $\mu_{r}<e_{r}<\tau_{1}<\nu_{\mu}$ & 2.81 \\
$\operatorname{nuSP}_{5}[\mu 2 \mathrm{~b}]$ & $\mu_{r}<e_{r}<\tau_{1}<\chi_{2}^{0}$ & 0.56 \\
$\operatorname{nuSP}_{5}[\mu 3]$ & $\mu_{r}<e_{r}<\chi_{2}^{0}<\chi_{1}^{ \pm}$ & 0.71 \\
\hline $\operatorname{nuSP}_{5}[\mu 4]$ & $\mu_{r}<e_{r}<\nu_{e}<\nu_{\mu}$ & 0.04 \\
\hline $\operatorname{nuSP}_{5}[\mu 5]$ & $\mu_{r}<\tau_{1}<e_{r}<\nu_{\mu}$ & 0.04 \\
\hline $\operatorname{nuSP}_{5}[\mathrm{~N} 1]$ & $\chi_{2}^{0}<\chi_{1}^{ \pm}<H^{0}<A^{0}$ & 0.90 \\
\hline $\operatorname{nuSP}_{5}[\mathrm{~N} 2]$ & $\chi_{2}^{0}<\chi_{1}^{ \pm}<\tau_{1}<H^{0}$ & 0.49 \\
\hline $\operatorname{nuSP}_{5}[\mathrm{~N} 3]$ & $\chi_{2}^{0}<\chi_{1}^{ \pm}<\mu_{r}<e_{r}$ & 0.04 \\
\hline
\end{tabular}

\begin{tabular}{|l|l|c|}
\hline Pattern Label & \multicolumn{1}{|c|}{ Mass Hierarchy } & $\%$ \\
\hline $\operatorname{nuSP}_{5}[\tau 1 \mathrm{a}]$ & $\tau_{1}<\chi_{1}^{ \pm}<\chi_{2}^{0}<\chi_{3}^{0}$ & 3.04 \\
$\operatorname{nuSP}_{5}[\tau 1 \mathrm{~b}]$ & $\tau_{1}<\chi_{1}^{ \pm}<\chi_{2}^{0}<\nu_{\tau}$ & 0.07 \\
$\operatorname{nuSP}_{5}[\tau 1 \mathrm{c}]$ & $\tau_{1}<\chi_{1}^{ \pm}<\chi_{2}^{0}<H^{0}$ & 0.04 \\
\hline $\operatorname{nuSP}_{5}[\tau 2 \mathrm{a}]$ & $\tau_{1}<\mu_{r}<e_{r}<\chi_{2}^{0}$ & 4.39 \\
$\operatorname{nuSP}_{5}[\tau 2 \mathrm{~b}]$ & $\tau_{1}<\mu_{r}<e_{r}<\nu_{\mu}$ & 3.90 \\
$\operatorname{nuSP}_{5}[\tau 2 \mathrm{c}]$ & $\tau_{1}<\mu_{r}<e_{r}<\nu_{\tau}$ & 0.49 \\
$\operatorname{nuSP}_{5}[\tau 2 \mathrm{~d}]$ & $\tau_{1}<\mu_{r}<e_{r}<t_{1}$ & 0.04 \\
\hline $\operatorname{nuSP}_{5}[\tau 3 \mathrm{a}]$ & $\tau_{1}<\chi_{2}^{0}<\chi_{1}^{ \pm}<H^{0}$ & 3.07 \\
$\operatorname{nuSP}_{5}[\tau 3 \mathrm{~b}]$ & $\tau_{1}<\chi_{2}^{0}<\chi_{1}^{ \pm}<\mu_{r}$ & 1.12 \\
$\operatorname{nuSP}_{5}[\tau 3 \mathrm{c}]$ & $\tau_{1}<\chi_{2}^{0}<\chi_{1}^{ \pm}<\nu_{\tau}$ & 1.05 \\
$\operatorname{nuSP}_{5}[\tau 3 \mathrm{~d}]$ & $\tau_{1}<\chi_{2}^{0}<\chi_{1}^{ \pm}<\chi_{3}^{0}$ & 0.19 \\
$\operatorname{nuSP}_{5}[\tau 3 \mathrm{e}]$ & $\tau_{1}<\chi_{2}^{0}<\chi_{1}^{ \pm}<t_{1}$ & 0.15 \\
\hline $\operatorname{nuSP}_{5}[\tau 4 \mathrm{a}]$ & $\tau_{1}<\nu_{\tau}<\tau_{2}<\chi_{2}^{0}$ & 0.19 \\
$\operatorname{nuSP}_{5}[\tau 4 \mathrm{~b}]$ & $\tau_{1}<\nu_{\tau}<\tau_{2}<\chi_{1}^{ \pm}$ & 0.04 \\
\hline $\operatorname{nuSP}_{5}[\tau 5]$ & $\tau_{1}<\nu_{\tau}<\chi_{2}^{0}<\chi_{1}^{ \pm}$ & 0.07 \\
\hline $\operatorname{nuSP}_{5}[\tau 6]$ & $\tau_{1}<H^{0}<A^{0}<H^{ \pm}$ & 0.07 \\
\hline $\operatorname{nuSP}_{5}[\tau 7]$ & $\tau_{1}<t_{1}<\nu_{\tau}<\tau_{2}$ & 0.04 \\
\hline $\operatorname{nuSP}_{5}[\mathrm{t} 1]$ & $t_{1}<\chi_{1}^{ \pm}<\chi_{2}^{0}<\chi_{3}^{0}$ & 0.19 \\
\hline $\operatorname{nuSP}_{5}[\mathrm{t} 2]$ & $t_{1}<\tau_{1}<\chi_{2}^{0}<\chi_{1}^{ \pm}$ & 0.11 \\
\hline $\operatorname{nuSP}_{5}[\mathrm{t} 3]$ & $t_{1}<\tau_{1}<<\tau_{2}$ & 0.04 \\
\hline $\operatorname{nuSP}_{5}[\mathrm{t} 4]$ & $t_{1}<\tau_{1}<\chi_{1}^{ \pm}<\chi_{2}^{0}$ & 0.04 \\
\hline & & \\
\hline
\end{tabular}

Table 9. Sparticle mass hierarchies for the nuSUGRA light third generation case (Model [5]). The high scale parameters lie in the range $m_{0}^{(1)}=m_{0}^{(2)}=m_{0} \in[0.1,10] \mathrm{TeV}, m_{0}^{(3)}=\frac{m_{0}}{1 \mathrm{TeV}+m_{0}}$, $m_{1 / 2} \in[0.1,1.5] \mathrm{TeV}, \frac{A_{0}}{m_{0}} \in[-5,5], \tan \beta \in[2,50], \mu>0$, with the constraints $\Omega h^{2}<0.12$, $m_{h^{0}}>120 \mathrm{GeV}$.

analysis of the sparticle mass hierarchies for the nuSUGRA Model [4] with nonuniversality in the Higgs boson mass sectors, and in table 9 an analysis is given of the sparticle mass hierarchies for nuSUGRA Model [5] for the case when nonuniversality is in the third generation sfermion sector. In table 10 a number of benchmarks are presented for both universal and nonuniversal SUGRA cases. These benchmarks respect all the collider, flavor and cosmological constraints and are not excluded by the by Run-I of the LHC.

\section{Sparticle mass hierarchies and simplified models}

Recently a new avenue for the exploration of new physics at colliders has been explored via the so-called simplified models [32-35, 37-40]. For example, one might consider a system 


\begin{tabular}{|c||c|c|c|c||c|c|c|c||c|c|}
\hline $\begin{array}{c}\text { SUGRA } \\
\text { Hierarchy }\end{array}$ & $\begin{array}{c}m_{0} \\
(\mathrm{GeV})\end{array}$ & $\begin{array}{c}m_{1 / 2} \\
(\mathrm{GeV})\end{array}$ & $\begin{array}{c}A_{0} \\
(\mathrm{GeV})\end{array}$ & $\begin{array}{c}\tan \beta \\
\left(\frac{v_{2}}{v_{1}}\right)\end{array}$ & $\delta_{M_{2}}$ & $\delta_{M_{3}}$ & $\left(\delta_{H_{1}}, \delta_{H_{2}}\right)$ & $\delta_{M_{q_{3}}}$ & $\begin{array}{c}\mu \\
(\mathrm{GeV})\end{array}$ & $\begin{array}{c}m_{h^{0}} \\
(\mathrm{GeV})\end{array}$ \\
\hline $\mathrm{mSP}[\mathrm{C} 1 \mathrm{a}]$ & 6183 & 470 & -4469 & 52 & - & - & - & - & 269 & 126.1 \\
$\left.\mathrm{mSP}_{\mathrm{CC}} \mathrm{C}\right]$ & 3715 & 1080 & 706 & 52 & - & - & - & - & 569 & 123.1 \\
$\operatorname{nuSP}_{2}[\mathrm{C} 1 \mathrm{a}]$ & 5464 & 1049 & 4845 & 52 & -0.063 & - & - & - & 583 & 124.0 \\
$\operatorname{nuSP}_{2}[\mathrm{C} 2 \mathrm{a}]$ & 2005 & 1234 & -3105 & 32 & -0.446 & - & - & - & 1999 & 125.4 \\
$\operatorname{nuSP}_{3}[\mathrm{Cla}]$ & 5446 & 500 & -3940 & 24 & - & -0.524 & - & - & 285 & 125.3 \\
$\operatorname{nuSP}_{3}[g 1 \mathrm{a}]$ & 5019 & 846 & 7759 & 15 & - & -0.819 & - & - & 2066 & 123.4 \\
$\operatorname{nuSP}_{4}[\mathrm{C} 1 \mathrm{a}]$ & 1210 & 848 & -1656 & 26 & - & - & $(-0.830,-1.205)$ & - & 571 & 123.5 \\
$\operatorname{nuSP}_{4}[\tau 2 \mathrm{a}]$ & 591 & 901 & -1746 & 31 & - & - & $(-2.089,-6.704)$ & - & 1419 & 123.6 \\
$\operatorname{nuSP}_{5}[\mathrm{C} 1 \mathrm{a}]$ & 2007 & 1155 & -989 & 48 & - & - & - & -0.361 & 589 & 123.3 \\
$\operatorname{nuSP}_{5}[\mathrm{C} 2 \mathrm{a}]$ & 2301 & 1241 & -2185 & 31 & - & - & - & -0.584 & 541 & 126.0 \\
\hline
\end{tabular}

Table 10. Benchmarks are given for SUGRA Models [1]-[5]. The sparticle mass hierarchy is specified for each benchmark. These particular model points are chosen due to having a mass pattern which has an especially large percentage of occurrence and also having passed collider, flavor and cosmological constraints. Further, they satisfy $\left(m_{\mathrm{NLSP}}+m_{\mathrm{NNLSP}}\right) / 2<600 \mathrm{GeV}$ and have a maximum NLSP-LSP mass gap. The nonuniversalities are defined as the following: $M_{2}=$ $m_{1 / 2}\left(1+\delta_{M_{2}}\right)$ for Model [2], $M_{3}=m_{1 / 2}\left(1+\delta_{M_{3}}\right)$ for Model [3], $m_{H_{i}}^{2}=m_{0}^{2}\left(1+\delta_{H_{i}}\right)$, where $i=1,2$ for Model [4] and $m_{q_{3}}=m_{0}\left(1+\delta_{m_{q_{3}}}\right)$ for Model [5].

of three particles: $A, B, C$ with masses $m_{A}, m_{B}, m_{C}$ and the hierarchy

$$
m_{A}>m_{B}>m_{C}
$$

One further assumes that the branching ratio of the decay of $A$ into the state $B$ is $100 \%$, and likewise the branching ratio of $B$ to $C$ is also $100 \%$. More generally, one could also have $A$ going directly to $C$, but often in simplified models the direct decay of $A$ to $C$ is ignored. One could also consider simplified models including four particles $A, B, C, D$ with the mass hierarchy

$$
m_{A}>m_{B}>m_{C}>m_{D} .
$$

Here one has six allowed branchings $A \rightarrow B, C, D, B \rightarrow C, D$ and $C \rightarrow D$. Again the simplified assumption would be to consider just a cascade type decay $A \rightarrow B \rightarrow C \rightarrow D$ which involves only three branchings which can all be assumed to be $100 \%$. A specific example of a three particle simplified model is given by the decay chain $\chi_{2}^{0} \rightarrow \chi_{1}^{ \pm} \rightarrow \chi_{1}^{0}$, and examples of four and five particles simplified models are given by the decay chains $\widetilde{g} \rightarrow \chi_{2}^{0} \rightarrow \chi_{1}^{ \pm} \rightarrow \chi_{1}^{0}$ and $\chi_{3}^{0} \rightarrow \widetilde{g} \rightarrow \chi_{2}^{0} \rightarrow \chi_{1}^{ \pm} \rightarrow \chi_{1}^{0}$.

In simplified models one makes an ad hoc choice of the lightest particles and their decay chains. However, a simplified model with particles chosen in an ad hoc fashion may not be embeddable in a high scale model. Thus, it is worthwhile to investigate the classes of simplified models that can arise from truncation of sparticle mass hierarchies that arise from a high scale model. In this way one can work with simplified models at the level of a preliminary investigation keeping in mind that it is a truncation of a UV complete model which would eventually replace the simplified model by a more complete one. In this work we give a fairly exhaustive analysis of the class of simplified models that arise in truncation of sparticle mass hierarchies in supergravity unified models using universal as 


\begin{tabular}{|c|c|c|c|c|c|c|}
\hline $\mathrm{mSP}[\mathrm{C} 2]$ & $\mathrm{mSP}[\mathrm{C} 3]$ & $\underline{\operatorname{mSP}}[\tau 4]$ & $\operatorname{nuSP}_{3}[t 6 a]$ & $\operatorname{nuSP}_{3}[t 4]$ & $\operatorname{nuSP}_{3}[g 3]$ & $\operatorname{nuSP}_{3}[\tau 4]$ \\
\hline$A^{0}$ & $\chi_{3}^{0}$ & $\chi_{1}^{ \pm}$ & $\chi_{2}^{0}$ & $\chi_{1}^{ \pm}$ & $\chi_{1}^{ \pm}$ & $\boldsymbol{H}^{ \pm}$ \\
\hline$H^{0}$ & $\tilde{g}$ & $\chi_{2}^{0}$ & $\chi_{1}^{ \pm}$ & $\chi_{2}^{0}$ & $\chi_{2}^{0}$ & $A^{0}$ \\
\hline$\chi_{2}^{0}$ & $\chi_{2}^{0}$ & $\tilde{\boldsymbol{t}}_{1}$ & $\tilde{\tau}_{1}$ & $\tilde{g}$ & $\tilde{t}_{1}$ & $H^{0}$ \\
\hline$\chi_{1}^{ \pm}$ & $\chi_{1}^{ \pm}$ & $\tilde{\tau}_{1}$ & $\tilde{t}_{1}$ & $\tilde{t}_{1}$ & $\tilde{g}$ & $\tilde{\tau}_{1}$ \\
\hline$\chi_{1}^{0}$ & $\chi_{1}^{0}$ & $\chi_{1}^{0}$ & $\chi_{1}^{0}$ & $\chi_{1}^{0}$ & $\chi_{1}^{0}$ & $\chi_{1}^{0}$ \\
\hline
\end{tabular}

Figure 1. An illustration of simplified models with five lightest particles arising from SUGRA unified models where the masses are in ascending order. Simplified models with a lower number of particles can be obtained by retaining the appropriate lower number of particles in the mass hierarchy. A more complete set of three, four and five particle simplified models can be obtained by retaining three, four and five particles in table 5 and in tables 6 to 9 .

well as nonuniversal boundary conditions, i.e., in mSUGRA as well as in nuSUGRA models as discussed in section $2 .{ }^{6}$ The results of the mass hierarchies are given in table 5 for the mSUGRA case and in tables 6 to 9 for the nonuniversal SUGRA case. Thus, table 5 gives the mass hierarchies that arise for five particles including the LSP. These can be truncated to give mass hierarchies for just three particle mass hierarchies.

An illustration of several simplified models arising from mSUGRA and from nonuniversal SUGRA models is given in figure 1 where the masses of the particles are in ascending order. One can use each of the columns to generate three, four or five particle simplified models. Most of the current work centers around keeping just three particles in the analysis. Such an approximation is valid if the mass gaps between the first three and the remaining higher ones are appreciable so that their inclusion would not radically change the signature calculus. However, often this is not the case. In mSUGRA, often $\chi_{1}^{ \pm}$and $\chi_{2}^{0}$ are essentially degenerate, as are $H^{0}, A^{0}, H^{ \pm}$; thus, a truncation will lead to significant errors. Additionally, if there is a strongly interacting particle lying just above the lowest three, a neglect of this particle will also lead to erroneous results.

We discuss further the relative advantages and disadvantages of the simplified models. In the analysis of the simplified models, the coupling and the masses of the particles can be taken to be adjustable parameters. This procedure circumvents carrying out radiative breaking of the electroweak symmetry every time the parameters are varied. Here one already has the masses and the couplings and so one can get down to the task of fitting the data. Further, the simplified models can explore the phase space of signatures which may otherwise be forbidden from the point of view of well-motivated models with constraints such as, for example, the constraint of radiative breaking of the electroweak symmetry. However, the disadvantage is that some of the models may not be embeddable in a UV complete theory and the connection of such models with fundamental physics one is trying to explore becomes tenuous. On the other hand, if one uses the simplified models arising

\footnotetext{
${ }^{6}$ Some of the preliminary results were presented at SUSY2014 [64].
} 
from the supergravity unified models, the connection with a high scale theory is much stronger.

While the simplified models are much easier to deal with, the approximation of using just three particles can be very limiting as noted earlier. Thus, for example, for the case where several particles are clustered, the approximation of just keeping three lowest ones is not justified. An example of this is given in figure 2 where the electroweak gauginos are packed closely together. Also as noted above, if there is a strongly interacting particle in the vicinity, then an analysis that ignores it would lead to erroneous results. Additionally, of course any randomly chosen three light particles would not necessarily arise from the spectrum of a UV complete theory.

The observant reader may have noticed that in table 5 as well in tables 6 to 9 the first five particles are mostly electroweak. The colored particles when present appear with low frequency as given by the percentage of occurrence shown in the last column of the tables. The reason for this phenomenon can be traced in part to the largeness of the Higgs mass and in part to the lower experimental limit on the gluino mass of around $1 \mathrm{TeV}$. Thus the Higgs boson mass of $\sim 125-126 \mathrm{GeV}$ requires that the average SUSY scale $M_{s} \sim \sqrt{m_{\tilde{t}_{1}} m_{\tilde{t}_{2}}}$ be in the few $\mathrm{TeV}$ range to produce the loop correction necessary to raise the Higgs boson mass from the tree value of $\leq M_{Z}$ to the experimentally observed value. In mSUGRA a large $M_{s}$ can arise from either a large $m_{0}$ or a large gluino mass or by a combination of both. For large $m_{0}$ all the squarks and sleptons except for the possibility of a light stop will be above the $\mathrm{TeV}$ region. Combined with the current experimental lower limit on the gluino mass of roughly a $\mathrm{TeV}$, these constraints imply that all of the colored particles except for the possibility of a light stop or gluino will not populate the lightest set of sparticle patterns. There is, however, an alternate possibility of generating a large $M_{s}$ needed for producing the large loop correction to lift the tree level Higgs boson mass. Here one can choose $m_{0}$ to be in the low $\mathcal{O}(100 \mathrm{GeV})$ region, and choose the gluino mass at the GUT scale to be in the several $\mathrm{TeV}$ region [21]. In this case the $\mathrm{RG}$ running drives the squark masses to high values while the slepton masses are low since they do not receive large corrections from RG evolution. In each of these cases one finds that the low mass sparticle spectrum will contain mostly the electroweak particles and color particles would be rare, often consisting of just the stop or the gluino consistent with the experimental lower mass limits. The result of this analysis obviously has important implications regarding sparticle searches, i.e., the focus of the searches be geared to look for the electroweak particles and in the color sector for the stop and the gluino which are often the lightest colored particles.

\section{Model signatures}

The most effective signatures for the LHC Run-II will be correlated strongly with the type of mass hierarchical patterns considered. Below we discuss a few illustrative examples and the possible signatures associated with the chosen patterns. We consider first the sparticle production for the hierarchical patterns $\mathrm{mSP}[\mathrm{C} 1]-\mathrm{mSP}[\mathrm{C} 4]$. If we retain only the three lightest particles then the patterns $\mathrm{mSP}[\mathrm{C} 1]-\mathrm{mSP}[\mathrm{C} 4]$ are indistinguishable. To discriminate among the subcases, for example, between $\mathrm{mSP}[\mathrm{C} 1]$ and $\mathrm{mSP}[\mathrm{C} 2]$ we need to 


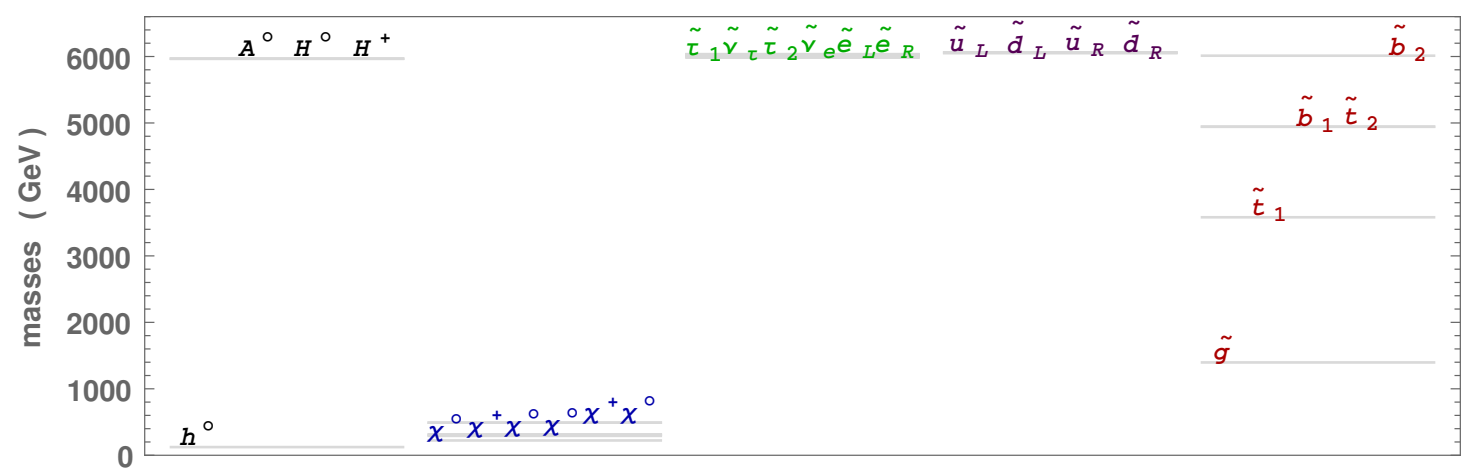

Figure 2. An exhibition of clustering of particles arising from a high scale model where the inputs for the model point are $m_{0}=6038 \mathrm{GeV}, m_{1 / 2}=538 \mathrm{GeV}, A_{0}=-2734 \mathrm{GeV}, \tan \beta=10$ and $\mu$ is positive. The particles are arranged according to their masses as indicated by the y-axis.

consider at least a four particle mass hierarchy. For both of these patterns the first three particles have the hierarchy $\chi_{1}^{0}<\chi_{1}^{ \pm}<\chi_{2}^{0}$ but differ in the placement of the 4 th lightest particle. For $\mathrm{mSP}[\mathrm{C} 1]$ it is $\chi_{3}^{0}$, while for $\mathrm{mSP}[\mathrm{C} 2]$ it is $H^{0}$. A similar situation arises for the hierarchical mass patterns nuSP $2[\mathrm{C} 1]-$ nuSP $_{5}[\mathrm{C} 5]$. Here also these five patterns can only be discriminated by considering more than three particles. Further, the only difference between $\mathrm{mSP}[\mathrm{C} 1]-\mathrm{mSP}[\mathrm{C} 4]$ vs $\operatorname{nuSP}_{2}[\mathrm{C} 1]-\operatorname{nuSP}_{2}[\mathrm{C} 4]$ relates to the mass gaps between the three sparticle states. If we keep only the first three particles in the mass hierarchies, then the particles likely to be produced at the colliders for the patterns $\mathrm{mSP}[\mathrm{C} 1]-\mathrm{mSP}[\mathrm{C} 4]$ and $\mathrm{nuSP}_{2}[\mathrm{C} 1]-\mathrm{nuSP}_{2}[\mathrm{C} 5]$ are as follows:

$$
\mathrm{mSP}[\mathrm{C} 1]-\mathrm{mSP}[\mathrm{C} 4] / \mathrm{nuSP}_{2}[\mathrm{C} 1]-\operatorname{nuSP}_{2}[\mathrm{C} 5]: \chi_{1}^{ \pm} \chi_{1}^{\mp}, \quad \chi_{1}^{ \pm} \chi_{1}^{0}, \quad \chi_{1}^{0} \chi_{2}^{0}, \quad \chi_{1}^{ \pm} \chi_{2}^{0}, \quad \chi_{2}^{0} \chi_{2}^{0} .
$$

These sparticle pairs would arise from the parton-level processes $q \bar{q} \rightarrow \chi_{i}^{ \pm} \chi_{j}^{\mp}, \chi_{k}^{0} \chi_{\ell}^{0}$, and $u \bar{d} \rightarrow \chi_{i}^{+} \chi_{k}^{0}$ etc. The chargino can decay so that $\chi_{1}^{-} \rightarrow W^{-} \chi_{1}^{0}$ with $W^{-} \rightarrow \ell^{-} \bar{\nu}$ so we will have $\ell^{-}+E_{T}^{\text {miss }}$ in the decay of the chargino. Thus, $\chi_{1}^{ \pm} \chi_{1}^{0}$ will produce a charged lepton and missing energy. Next $\chi_{1}^{+} \chi_{1}^{-}$will produce two charged leptons and $E_{T}^{\text {miss }}$. Since a chargino can also decay via a $W^{*}$ into $q_{1} \bar{q}_{2}+\chi_{1}^{0}$, we can have in addition a single charged lepton plus jets and $E_{\mathrm{T}}^{\mathrm{miss}}$. Now $\chi_{2}^{0}$ will have decays such as $\ell^{+} \ell^{-}+E_{\mathrm{T}}^{\mathrm{miss}}$. Thus, $\chi_{1}^{ \pm}$ $\chi_{2}^{0}$ will have trileptonic decays [65-68] $\ell_{1}^{ \pm} \ell_{2}^{+} \ell_{2}^{-}+E_{\mathrm{T}}^{\text {miss }}$. The production channels $\chi_{1}^{0} \chi_{2}^{0}$ will have decays of the type $\ell^{+} \ell^{-}+E_{\mathrm{T}}^{\text {miss }}$ and $\chi_{2}^{0} \chi_{2}^{0}$ will have 4-lepton decays $\ell_{1}^{+} \ell_{1}^{-} \ell_{2}^{+} \ell_{2}^{-}+$ $E_{\mathrm{T}}^{\mathrm{miss}}$ as well as decays of the type $\ell^{+} \ell^{-}+$jets $+E_{\mathrm{T}}^{\text {miss }}$. For cases where the $\chi_{2}^{0}$ mass is large enough so that $m_{\chi_{2}^{0}}>m_{\chi_{1}^{0}}+m_{h^{0}}$, we can have on-shell decays $\chi_{2}^{0} \rightarrow \chi_{1}^{0} h^{0}$, and $h^{0}$ predominantly decays via the mode $h^{0} \rightarrow b \bar{b}$. This gives rise to important new signatures such as $\chi_{1}^{ \pm} \chi_{2}^{0} \rightarrow \ell^{ \pm} b \bar{b}+E_{\mathrm{T}}^{\mathrm{miss}}$ and $\chi_{2}^{0} \chi_{2}^{0} \rightarrow \ell^{+} \ell^{-} b \bar{b}$.

Next we consider the pattern nuSP ${ }_{2}[\mathrm{C} 6]$ and nuSP $2[\mathrm{C} 7]$ which have the same lowest three particle pattern, i.e., $\chi_{1}^{0}, \chi_{1}^{ \pm}, \tilde{\tau}_{1}$ with the mass hierarchy $m_{\chi_{1}^{0}}<m_{\chi_{1}^{ \pm}}<m_{\tilde{\tau}_{1}}$. Again to distinguish between nuSP${ }_{2}[\mathrm{C} 6]$ and nuSP${ }_{2}[\mathrm{C} 7]$ we would need to consider a four particle mass hierarchy. For these mass patterns we can produce a chargino-neutralino, two charginos or two staus in $p p$ collisions:

$$
\operatorname{nuSP}_{2}[\mathrm{C} 6], \operatorname{nuSP}_{2}[\mathrm{C} 7]: \chi_{1}^{ \pm} \chi_{1}^{0}, \chi_{1}^{+} \chi_{1}^{-}, \widetilde{\tau}_{1} \widetilde{\tau}_{1}^{*} .
$$


The signatures arising from $\chi_{1}^{ \pm} \chi_{1}^{0}$ and $\chi_{1}^{+} \chi_{1}^{-}$production are as discussed for mSP[C1]-

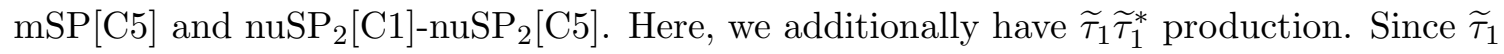
has the decay $\widetilde{\tau}_{1} \rightarrow \tau \chi_{1}^{0}$, we will have a signature of the type $\tau^{+} \tau^{-}+E_{\mathrm{T}}^{\mathrm{miss}}$. Additionally, since $\widetilde{\tau}_{1}$ is heavier than the chargino, we will have the decay $\widetilde{\tau}_{1}^{-} \rightarrow \chi_{1}^{-} \nu_{\tau}$ with $\chi_{1}^{-} \rightarrow \ell^{-} \bar{\nu}$. This will lead to signatures such as $\ell_{i}^{+} \ell_{j}^{-}+E_{\mathrm{T}}^{\text {miss }}$ where $\ell_{i}=e, \mu, \tau$. Closely related to the signatures arising from nuSP ${ }_{2}[\mathrm{C} 6]$ and nuSP $2[\mathrm{C} 7]$ are the signatures arising from nuSP $2[\tau 2]$ and nuSP $2[\tau 3]$ where we have the same three particles but the mass hierarchy is inverted for the top two, i.e., we $m_{\chi_{1}^{0}}<m_{\widetilde{\tau}_{1}}<m_{\chi_{1}^{ \pm}}$. Thus, the final states produced for these patterns will be the same as in $\operatorname{nuSP}_{2}[\mathrm{C} 6]$ and nuSP $2[\mathrm{C} 7]$ except that here the chargino is heavier than the stau. Thus, instead of stau decay into a chargino we have a chargino decaying into a stau which gives $\chi_{1}^{-} \rightarrow \widetilde{\tau}_{1}^{-} \bar{\nu}_{\tau}$.

Next we consider the pattern nuSP $2[\mathrm{C} 9]$ where the three lightest particles are $\chi_{1}^{0}, \chi_{1}^{ \pm}, \widetilde{t}_{1}$ with the mass hierarchy $m_{\chi_{1}^{0}}<m_{\chi_{1}^{ \pm}}<m_{\widetilde{t}_{1}}$. The sparticle states produced in $p p$ collisions are as follows

$$
\operatorname{nuSP}_{2}[\mathrm{C} 9]: \chi_{1}^{ \pm} \chi_{1}^{0}, \chi_{1}^{+} \chi_{1}^{-}, \widetilde{t}_{1} \widetilde{t}_{1}^{*} .
$$

The new production mode here is $\widetilde{t}_{1} \widetilde{t}_{1}^{*}$, where $\widetilde{t}_{1}$ has the decay channels

$$
\widetilde{t}_{1} \rightarrow t \chi_{1}^{0}, b \chi_{1}^{ \pm}, c \chi_{1}^{0} .
$$

Further, the chargino will have the decay $\chi_{1}^{ \pm} \rightarrow W^{ \pm} \chi_{1}^{0} \rightarrow \ell \nu \chi_{1}^{0}, j j \chi_{1}^{0}$ and the top quark has the decays

$$
t \rightarrow j j b, \ell \nu b .
$$

Thus $\widetilde{t}_{1} \widetilde{t}_{1}^{*}$ production will lead to a variety of signals involving leptons and jets and missing energy such as the signals $\ell^{+} \ell^{-} b \bar{b}+E_{\mathrm{T}}^{\text {miss }}, \ell b \bar{b}+$ jets $+E_{\mathrm{T}}^{\text {miss }}$, etc.

Another illustrative example is the mass pattern $\operatorname{nuSP}_{3}[g 1]$ where the lightest three particles are $\chi_{1}^{0}, \widetilde{g}$, and $\chi_{2}^{0}$, with the mass hierarchy $m_{\chi_{1}^{0}}<m_{\widetilde{g}}<m_{\chi_{2}^{0}}$. The sparticles states produced in $p p$ collisions consist of the following

$$
\operatorname{nuSP}_{3}[g 1]: \widetilde{g} \widetilde{g}, \quad \chi_{2}^{0} \chi_{2}^{0} .
$$

The new production mode here is $\widetilde{g} \widetilde{g}$ which will dominate the signatures since the gluino interacts strongly and the production cross section for $\widetilde{g} \widetilde{g}$ will be much greater than that of electroweak gaugino production. The gluino will have the decay $\widetilde{g} \rightarrow \widetilde{t t_{1}^{*}}$ where the decays of $\widetilde{t}_{1}$ are given in eq. (4.1) and the decays of the top in eq. (4.2). These will generate a variety of signatures involving leptons, at least $2 b$-jets, light jets and missing energy.

An overall issue in the analysis of signatures concerns the NLSP and LSP mass difference. This mass difference determines the $p_{\mathrm{T}}$ of the jets and leptons and the $E_{\mathrm{T}}^{\text {miss }}$ in the NLSP decay. A small mass difference between the NLSP and the LSP will lead to softer jets and leptons and a small $E_{\mathrm{T}}^{\text {miss }}$ may not pass the cuts or be distinguishable from the background. However, there are a variety of other signatures that can be investigated. For recent analyses relating to sparticle signature identification at the upgraded LHC see [69, 70]. An interesting issue relates to how one may discriminate among "mirror patterns". Thus consider, for example, the third and the fourth columns of figure 1. Retaining only the three lowest mass particles, column three has the hierarchy $\chi_{1}^{0}<\widetilde{\tau}_{1}<\widetilde{t}_{1}$ 
while column four has the hierarchy $\chi_{1}^{0}<\widetilde{t}_{1}<\widetilde{\tau}_{1}$. Thus aside from the LSP the spectrum for column four is inverted relative to that for column three. In a similar fashion the lightest three particle spectrum arising from column five is $\chi_{1}^{0}<\tilde{t}_{1}<\widetilde{g}$ while that from column six is $\chi_{1}^{0}<\widetilde{g}<\widetilde{t}_{1}$. One may call such pairs mirror patterns. It should be interesting to investigate the characteristic signals that can discriminate between the two mirror patterns.

As mentioned already, a study of signatures based on three lowest lying particles would not lift the degeneracy among those patterns which have the same three lowest mass particles and we would need to include higher lying particles to discriminate among the patterns. Further, as discussed in section 3 keeping just the three lowest lying particles is inadequate when there is a clustering of particles as illustrated in figure 2 . In this case, all particles within the cluster must be taken account of. Another example where truncation to three or four particles is inadequate is when there is a strongly interacting particle lying close above. In this case again one may be lead to erroneous results by the truncation procedure of constructing a simplified model.

\section{Benchmarks for future SUSY searches at colliders}

Benchmarks are useful as illustrative examples of signature analyses that can lead to new discovery channels for superpartner particles. Here we give a few benchmarks which satisfy all the current collider, flavor and cosmological constraints. Specifically, we impose the following set of constraints in choosing the benchmarks: $m_{h^{0}} \in[123,127] \mathrm{GeV}, \Omega_{\chi_{1}^{0}} h^{2}<$ $0.12, \mathcal{B} r\left(B_{s}^{0} \rightarrow \mu^{+} \mu^{-}\right)<6.2 \times 10^{-9}, \mathcal{B} r\left(B \rightarrow X_{s} \gamma\right)<4.27 \times 10^{-4}$. Additionally, we only selected benchmarks that have sparticle mass hierarchies that are frequently observed in the constrained parameter space. In table 10 we give a set of benchmarks for both universal and nonuniversal SUGRA cases. Here we also identify the corresponding SUGRA pattern to which they belong. The benchmarks that are displayed satisfy the LHC Run-I exclusion constraint in the $m_{0}-m_{1 / 2}$ plane. These benchmarks should be useful for future SUSY searches at colliders. An interesting feature of table 10 is that most of the benchmarks have relatively small $\mu$ compared to $m_{0}$ which points to the fact that they lie on the hyperbolic branch of radiative breaking of the electroweak symmetry [71-75] and are thus natural according to the criteria discussed in [71].

\subsection{Signature analysis for an mSUGRA benchmark}

First we consider a benchmark within mSUGRA. Our model's GUT scale parameters and observables are given in table 2. The particle mass hierarchy for this model point is $m_{\chi_{1}^{ \pm}}<m_{\chi_{2}^{0}}<m_{\chi_{3}^{0}}<m_{\chi_{4}^{0}}$. This benchmark has a light LSP of mass $m_{\chi_{1}^{0}}=199 \mathrm{GeV}$, a chargino NLSP of mass $m_{\chi_{1}^{ \pm}}=261 \mathrm{GeV}$ and a second neutralino of mass $m_{\chi_{2}^{0}}=271 \mathrm{GeV}$. The first and the second generation squarks are heavy, $m_{\widetilde{q}} \gtrsim 6 \mathrm{TeV}$, the stop and the sbottom are $m_{\widetilde{t}_{1}}=3.6 \mathrm{TeV}, m_{\widetilde{b}_{1}}=4 \mathrm{TeV}$, and a gluino at $m_{\widetilde{g}}=1257 \mathrm{GeV}$. With the light neutralinos/charginos and a relatively light gluino, the total SUSY cross section is $740 \mathrm{fb}$ at $14 \mathrm{TeV}$. Since the rest of the spectrum is heavy, $\chi_{1}^{ \pm}$and $\chi_{2}^{0}$ decay through an off shell $W$ and $Z$ generating relatively soft jets and leptons. 
To test the visibility of this model at $14 \mathrm{TeV}$, we study the trilepton final state and follow the ATLAS search [76]. This analysis is based on a simplified model with only $\chi_{1}^{0}$, $\chi_{2}^{0}$, and $\chi_{1}^{ \pm}$. It is optimized for $8 \mathrm{TeV}$ and should be re-tuned for $14 \mathrm{TeV}$, but we use similar cuts as our starting point. The ATLAS analysis defines the signal region SR0 $\tau$ a which is the most sensitive to $\chi_{1}^{ \pm}$and $\chi_{2}^{0}$ decays through $W$ and $Z$ bosons. This signal region requires 3 leptons ( $e$ or $\mu$ ) including a same flavor opposite sign (SFOS) pair. The SFOS pair giving the invariant mass closest to the $Z$ mass is then identified and the remaining lepton's momentum is used to calculate the transverse mass defined as

$$
m_{\mathrm{T}}^{2}\left(\vec{p}_{\mathrm{T}}^{\ell}, \vec{p}_{\mathrm{T}}^{\text {miss }}\right)=2 p_{\mathrm{T}}^{\ell} E_{\mathrm{T}}^{\text {miss }}-2 \vec{p}_{\mathrm{T}}^{\ell} \cdot \vec{p}_{\mathrm{T}}^{\text {miss }} .
$$

The main irreducible background for this channel is diboson ( $W Z$ and $Z Z$ ), $t \bar{t} V$ and $t Z$ productions. The reducible backgrounds include single and pair production of top quarks. We follow the ATLAS analysis and veto events with $b$-tagged jets to suppress the top quark production.

In a previous work on signature analysis by two of the authors (BA and PN) the standard model backgrounds at LHC energy of $\sqrt{s}=7 \mathrm{TeV}$ were generated in-house [77]. Fortunately, for the current analysis this computer intensive process was circumvented by employing the Snowmass Standard Model background [22] normalized to NLO and generated with five flavor MLM matching and in bins of $S_{\mathrm{T}}$ which is the scalar sum of $p_{\mathrm{T}}$ of all generator level particles. For signal event generation, we use PүтнiA [78] for hard scattering and showering/hadronization, and DELPHES [79] for detector simulation with the same card used in the Snowmass background that was tuned according to the detector performances in the last run. We normalize our signal cross section to match NLO cross section obtained by Prospino [80].

In the ATLAS analysis, the signal region SR0 $\tau$ a is composed of 20 disjoint bins with varying ranges of $m_{\mathrm{SFOS}}, m_{T}$ and $E_{T}^{\text {miss }}$. We follow a similar approach and simply study two of those bins that offer the best discrimination of signal from the SM background for our benchmark point. These are bin- 6 with cuts $m_{T}<80 \mathrm{GeV}, E_{\mathrm{T}}^{\text {miss }}>75 \mathrm{GeV}$ and bin-12 with cuts $m_{T}>110 \mathrm{GeV}, E_{\mathrm{T}}^{\text {miss }}>75 \mathrm{GeV}$. The distributions of $m_{\mathrm{SFOS}}$ in those bins are displayed in figure 3 prior to the cut on that variable. A further cut to constrain $m_{\text {SFOS }}$ into a mass window following the ATLAS analysis is also displayed. Our calculations show that the SUSY signal produced by our mSUGRA benchmark point will be discoverable at the LHC Run-II at $5 \sigma$ significance defined by $S / \sqrt{B}=5$ with an integrated luminosity of $L \gtrsim 340 \mathrm{fb}^{-1}\left(135 \mathrm{fb}^{-1}\right)$ by using the cuts of bin-6(12).

\subsection{Signature analysis for an nuSUGRA benchmark}

Next, we consider a nonuniversal SUGRA benchmark with nonuniversality in the gaugino sector as given in table 3. The particle mass hierarchy for this model point is $m_{\chi_{1}^{ \pm}}<$ $m_{\chi_{2}^{0}}<m_{\tilde{g}}<m_{\tilde{t}_{1}}$. Our benchmark has a neutralino LSP of mass $m_{\chi_{1}^{0}}=441 \mathrm{GeV}, \chi_{2}^{0}$ and $\chi_{1}^{ \pm}$are within $20 \mathrm{GeV}$ of the LSP, with a gluino of mass $1446 \mathrm{GeV}$ and a stop of mass $1481 \mathrm{GeV}$. These masses are beyond the current limits obtained by ATLAS and CMS experiments with simplified models. With a relatively light LSP and NLSP, this model can 

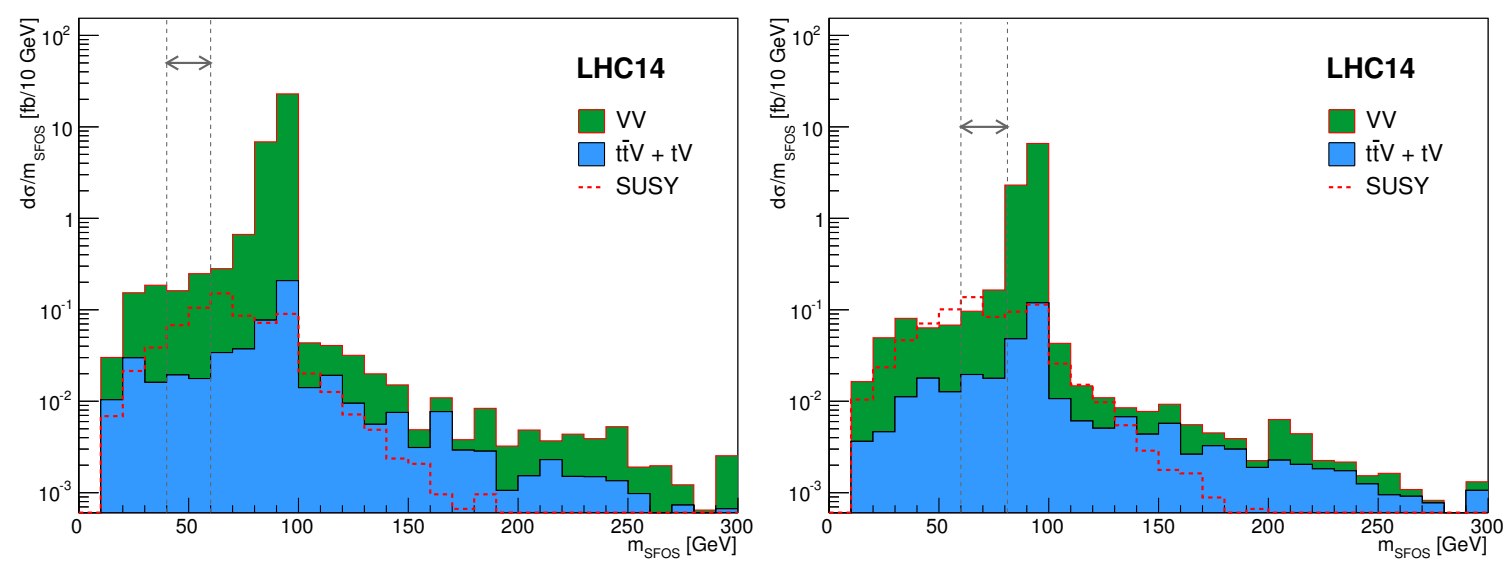

Figure 3. Distribution of $m_{\mathrm{SFOS}}$ in signal region SR0 $\tau$ a for bins 6 (on the left with cuts $m_{T}<$ $80 \mathrm{GeV}, E_{\mathrm{T}}^{\mathrm{miss}}>75 \mathrm{GeV}$ ) and 12 (on the right with cuts $m_{T}>110 \mathrm{GeV}, E_{\mathrm{T}}^{\text {miss }}>75 \mathrm{GeV}$ ) prior to the requirements on these variables. Main SM background consisting of $V V$ (green) and $t \bar{t} V+t V$ (green) are shown. The signal is shown in dashed red. The remaining invariant mass cuts used to define each signal region in the ATLAS analysis are also displayed. Our calculations show that $5 \sigma$ significance is obtained with an integrated luminosity of $L \gtrsim 340 \mathrm{fb}^{-1}$ (left) and $L \gtrsim 135 \mathrm{fb}^{-1}$ (right).

have considerable electroweak production of neutralinos and charginos. For example we obtain $\sigma\left(p p \rightarrow \chi_{2}^{0} \chi_{1}^{ \pm}\right)=69.7 \mathrm{fb}$ and $\sigma\left(p p \rightarrow \chi_{1}^{+} \chi_{1}^{-}\right)=33.2 \mathrm{fb}$ at NLO. But the small mass gap between LSP and NLSP/NNLSP make the final decay products quite soft resulting in some or most of them being missed by the triggers. One possibility of obtaining sufficiently energetic final states to pass the triggers is via a high- $p_{\mathrm{T}}$ ISR jet that will boost the event and provide large momenta to the final decay products. But with all these challenges, it will be quite difficult to observe this model at the LHC solely from electroweak production of neutralinos and charginos.

This model, however, has a gluino of mass $1446 \mathrm{GeV}$ which gives rise to a gluino pair production cross section of $\sigma(p p \rightarrow \widetilde{g} \widetilde{g})=23.0 \mathrm{fb}$ at NLO. Although this is smaller than the chargino-neutralino production cross section, the large mass gap provides energetic final states and large missing momentum which can easily get triggered. Since our squarks are all heavy except the stop, the gluino in this benchmark decays mostly into $t \bar{t} \chi_{1}^{0}$, producing 4 b-jets in the final state. To check the viability of detecting this benchmark at the LHC RunII, we considered the ATLAS analysis [81] for gluino pair production. Like the trilepton analysis we used for our mSUGRA benchmark model, this ATLAS analysis is also optimized for $8 \mathrm{TeV}$, but we use the exact same cuts as the ATLAS analysis [81] as our starting point. The ATLAS analysis introduces 9 signal regions demanding at least $4 / 6 / 7$ jets with at least 3 of them being tagged as a b-jet and 0 or 1 lepton. The main reducible background for this process is $t \bar{t}$ production where a c-jet or a hadronically decaying $\tau$ lepton is mis-tagged as a b-jet. The irreducible backgrounds from $t \bar{t}+b / b \bar{b}$ and $t \bar{t}+Z / h(\rightarrow b \bar{b})$ are dominant.

The following kinematic variables correlated with the overall mass scale are introduced: $H_{\mathrm{T}}^{4 \mathrm{j}}$ which is the scalar sum of the transverse momenta of the four leading jets, $m_{\mathrm{eff}}^{4 \mathrm{j}}$ which is the scalar sum of the $E_{\mathrm{T}}^{\text {miss }}$ and the transverse momenta of the four leading jets, 

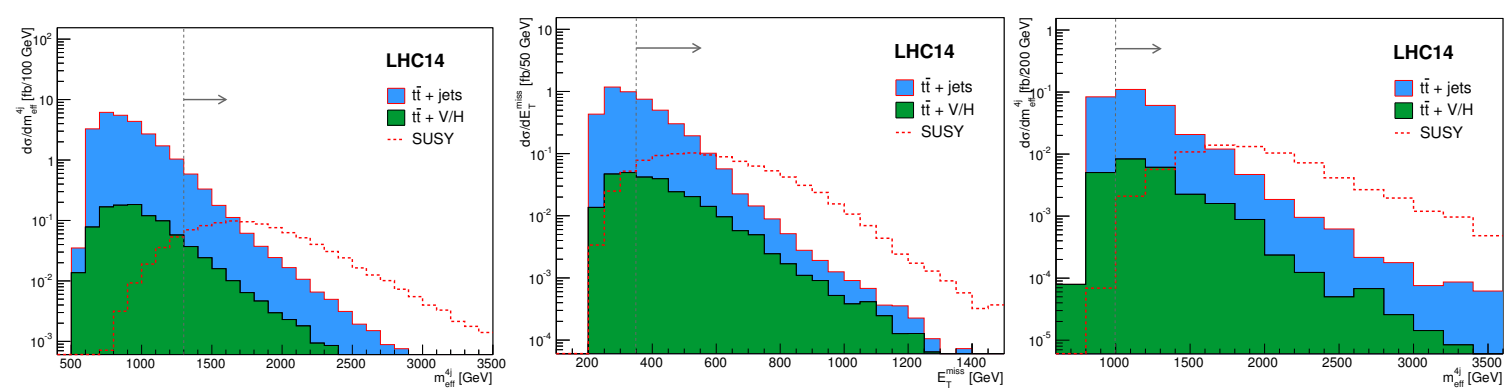

Figure 4. Distribution of $m_{\mathrm{eff}}^{4 \mathrm{j}}$ and $E_{\mathrm{T}}^{\mathrm{miss}}$ in the signal regions $\mathrm{SR}-0 \ell-4 \mathrm{j}-[\mathrm{A}, \mathrm{B}, \mathrm{C}]$ prior to the requirements on these variables. Arrows indicate the remaining cuts used to define the signal regions. Main SM background consisting of $t \bar{t}+$ jets (blue) and $t \bar{t}+V / H$ (green) are shown. The signal is shown in dashed red. Our calculations show that $5 \sigma$ significance is obtained with an integrated luminosity of $L \gtrsim 45 \mathrm{fb}^{-1}$ (left), $L \gtrsim 60 \mathrm{fb}^{-1}$ (middle) and $L \gtrsim 920 \mathrm{fb}^{-1}$ (right).

and $m_{\mathrm{eff}}^{\text {incl }}$ which is the scalar sum of $E_{\mathrm{T}}^{\text {miss }}$ and the transverse momenta of all jets with $p_{\mathrm{T}}>30 \mathrm{GeV}$. A cut on the minimum azimuthal separation between any of the four leading jets and the missing transverse momenta is also used to remove the multi-jet events. And finally transverse mass (defined in eq. (5.1)) computed from the leading lepton and the missing transverse momenta is used to cut down the $t \bar{t}$ events where one of the $W$ bosons decays leptonically. In the analysis we use the signal regions SR-0 $-4 \mathrm{j}-[\mathrm{A}, \mathrm{B}, \mathrm{C}], \mathrm{SR}-0 \ell-7 \mathrm{j}-$ $[\mathrm{A}, \mathrm{B}, \mathrm{C}]$ and SR-1 $\ell-6 \mathrm{j}-[\mathrm{A}, \mathrm{B}, \mathrm{C}]$ as defined by ATLAS in their analysis of SUSY signals at $\sqrt{s}=8 \mathrm{TeV}[81]$ and summarized in table 4 .

Distributions of $m_{\mathrm{eff}}^{4 \mathrm{j}}, m_{\mathrm{eff}}^{\mathrm{incl}}$ and $E_{\mathrm{T}}^{\mathrm{miss}}$ for all the signal regions SR-0 $\ell-4 \mathrm{j}-[\mathrm{A}, \mathrm{B}, \mathrm{C}], \mathrm{SR}-$ $0 \ell-7 \mathrm{j}-[\mathrm{A}, \mathrm{B}, \mathrm{C}]$ and $\mathrm{SR}-1 \ell-6 \mathrm{j}-[\mathrm{A}, \mathrm{B}, \mathrm{C}]$ are shown in figures 4 to 6 prior to the requirements on these variables. We follow the ATLAS analysis and apply all the cuts including the ones shown with arrows in the distributions and calculate the minimum integrated luminosities required for a $5 \sigma$ discovery. The analysis presented in figures 4 to 6 shows that for our benchmark model, almost all the signal regions are effective for discovery of sparticles at the LHC Run-II. Specifically we find that the signal regions SR-0 $\ell-4 \mathrm{j}-[\mathrm{A}, \mathrm{B}, \mathrm{C}]$ will require an integrated luminosity of $L \gtrsim 45 / 60 / 920 \mathrm{fb}^{-1}$, the signal regions SR-0 $\ell-7 \mathrm{j}$-[A,B,C] will require an integrated luminosity of $L \gtrsim 235 / 45 / 25 \mathrm{fb}^{-1}$, and the signal regions SR-1 $\ell-6 \mathrm{j}$ $[\mathrm{A}, \mathrm{B}, \mathrm{C}]$ will require an integrated luminosity of $L \gtrsim 510 / 265 / 160 \mathrm{fb}^{-1}$.

\section{Dark matter}

Each of the Models [1]-[5] in section 2 gives rise to spin-independent neutralino-proton cross sections which can have a significant range and, in some cases, span several orders of magnitude depending on the content of the neutralino being a bino, Higgsino, wino, or an admixture. The Higgs boson mass measurement is also a strong constraint on the spin-independent neutralino-proton cross section. In the left panel of figure 7 we display $R \times \sigma_{p \chi_{1}^{0}}^{\mathrm{SI}}$ vs the neutralino mass for mSUGRA Model [1] where $\sigma_{p \chi_{1}^{0}}^{\mathrm{SI}}$ is the spin-independent neutralino-proton cross section and $R=\rho_{\chi_{1}^{0}} / \rho_{c}$ where $\rho_{\chi_{1}^{0}}$ is the neutralino relic density and $\rho_{c}$ is the critical relic density needed to close the universe. The allowed parameter 

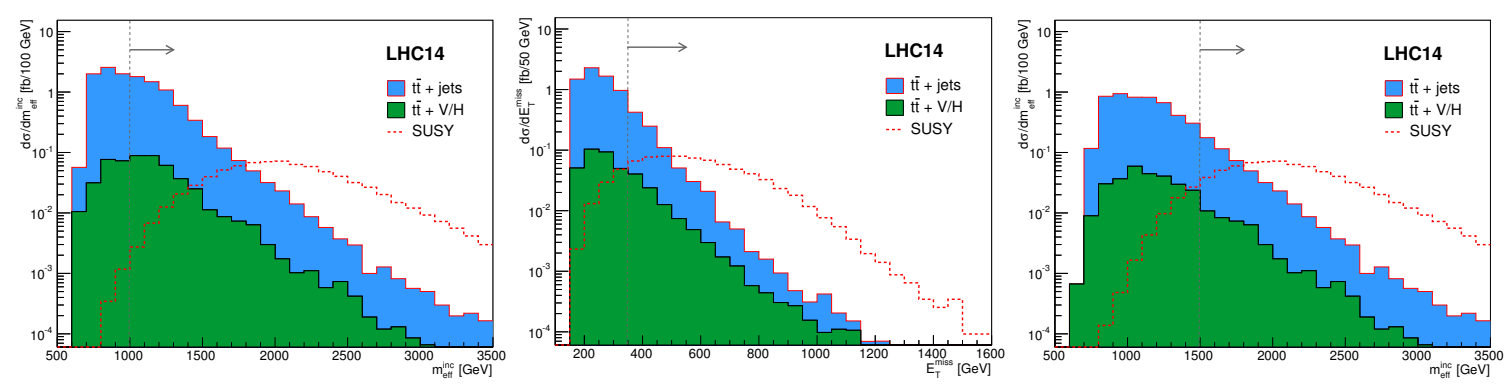

Figure 5. Distribution of $m_{\mathrm{eff}}^{\mathrm{incl}}$ and $E_{\mathrm{T}}^{\mathrm{miss}}$ in the signal regions SR-0 $\ell-7 \mathrm{j}-[\mathrm{A}, \mathrm{B}, \mathrm{C}]$ prior to the requirements on these variables. Arrows indicate the remaining cuts used to define the signal regions. Main SM background consisting of $t \bar{t}+$ jets (blue) and $t \bar{t}+V / H$ (green) are shown. The signal is shown in dashed red. Our calculations show that $5 \sigma$ significance is obtained with an integrated luminosity of $L \gtrsim 235 \mathrm{fb}^{-1}$ (left), $L \gtrsim 45 \mathrm{fb}^{-1}$ (middle) and $L \gtrsim 25 \mathrm{fb}^{-1}$ (right).
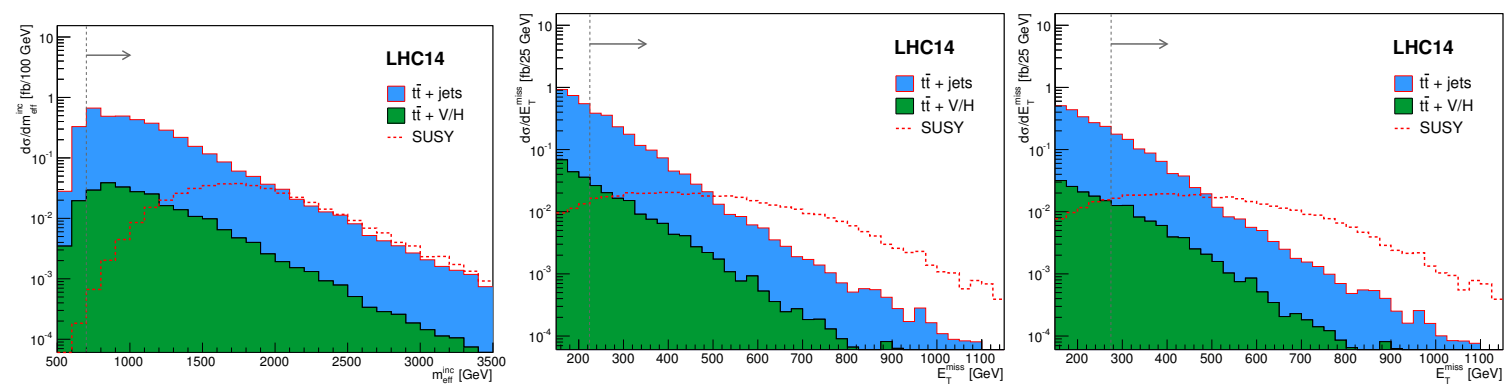

Figure 6. Distribution of $m_{\mathrm{eff}}^{\mathrm{incl}}$ and $E_{\mathrm{T}}^{\mathrm{miss}}$ in the signal regions SR-1 $\ell-6 \mathrm{j}-[\mathrm{A}, \mathrm{B}, \mathrm{C}]$ prior to the requirements on these variables. Arrows indicate the remaining cuts used to define the signal regions. Main SM background consisting of $t \bar{t}+$ jets (blue) and $t \bar{t}+V / H$ (green) are shown. The signal is shown in dashed red. Our calculations show that $5 \sigma$ significance is obtained with an integrated luminosity of $L \gtrsim 510 \mathrm{fb}^{-1}$ (left), $L \gtrsim 265 \mathrm{fb}^{-1}$ (middle) and $L \gtrsim 160 \mathrm{fb}^{-1}$ (right).

space in the $R \times \sigma_{p \chi_{1}^{0}}^{\mathrm{SI}}-m_{\chi_{1}^{0}}$ plane is colored according to the Higgs boson mass. In the right panel of figure 7 we give an analysis of $R \times \sigma_{p \chi_{1}^{0}}^{\mathrm{SI}}$ vs the neutralino mass for mSUGRA where we exhibit the sparticle mass patterns.

A very similar analysis for nuSUGRA Models is given in figures 8 to 11 . Thus in figure 8 an analysis is given of $R \times \sigma_{p, \chi_{1}^{0}}^{\mathrm{SI}}$ vs the neutralino mass for nuSUGRA Models [2][5] where the colors specify the Higgs boson mass. In figure 9 we give a composite of all cases considered, i.e., Models [1]-[5] with the additional constraint that $m_{h^{0}} \in[123,127] \mathrm{GeV}$, $\Omega_{\chi_{1}^{0}} h^{2}<0.12, \mathcal{B} r\left(B_{s}^{0} \rightarrow \mu^{+} \mu^{-}\right)<6.2 \times 10^{-9}, \mathcal{B} r\left(B \rightarrow X_{s} \gamma\right)<4.27 \times 10^{-4}$. In the figures here and also in later figures, we have exhibited the line where the signals from coherent scattering of solar, atmospheric and diffuse supernova neutrinos will begin to appear. The test of the neutrino cross sections is interesting in itself. However, for the purpose of WIMP detection the neutrino background must be subtracted or it would require a directional analysis in direct detection experiments to separate the neutrino backgrounds from the dark matter signals [82].

In figure 10 we give an analysis of the mass patterns for the nuSUGRA Models [2]-[3] and in figure 11 we give an analysis of mass patterns for the nuSUGRA models [4]-[5]. A 

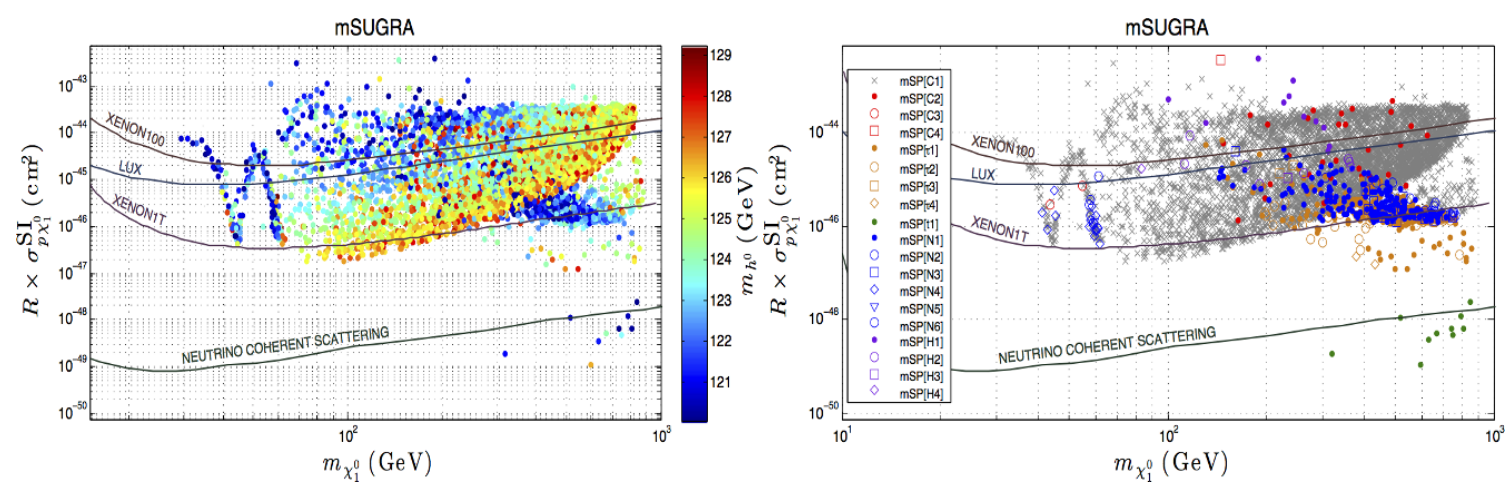

Figure 7. Left panel: a display of $R \times{\sigma_{p \chi_{1}^{0}}^{\mathrm{SI}}}^{\mathrm{a}}$ vs the neutralino mass for mSUGRA (Model [1]) where the colors exhibit the Higgs boson mass. Right panel: Exhibited are various hierarchical patterns contributing to the allowed parameter space where the constraints $\Omega h^{2}<0.12, m_{h^{0}}>120 \mathrm{GeV}$ hold. The analysis indicates that a knowledge of the spin-independent neutralino-proton cross section along with the neutralino mass will allow one to identify the possible underlying sparticle mass hierarchy.
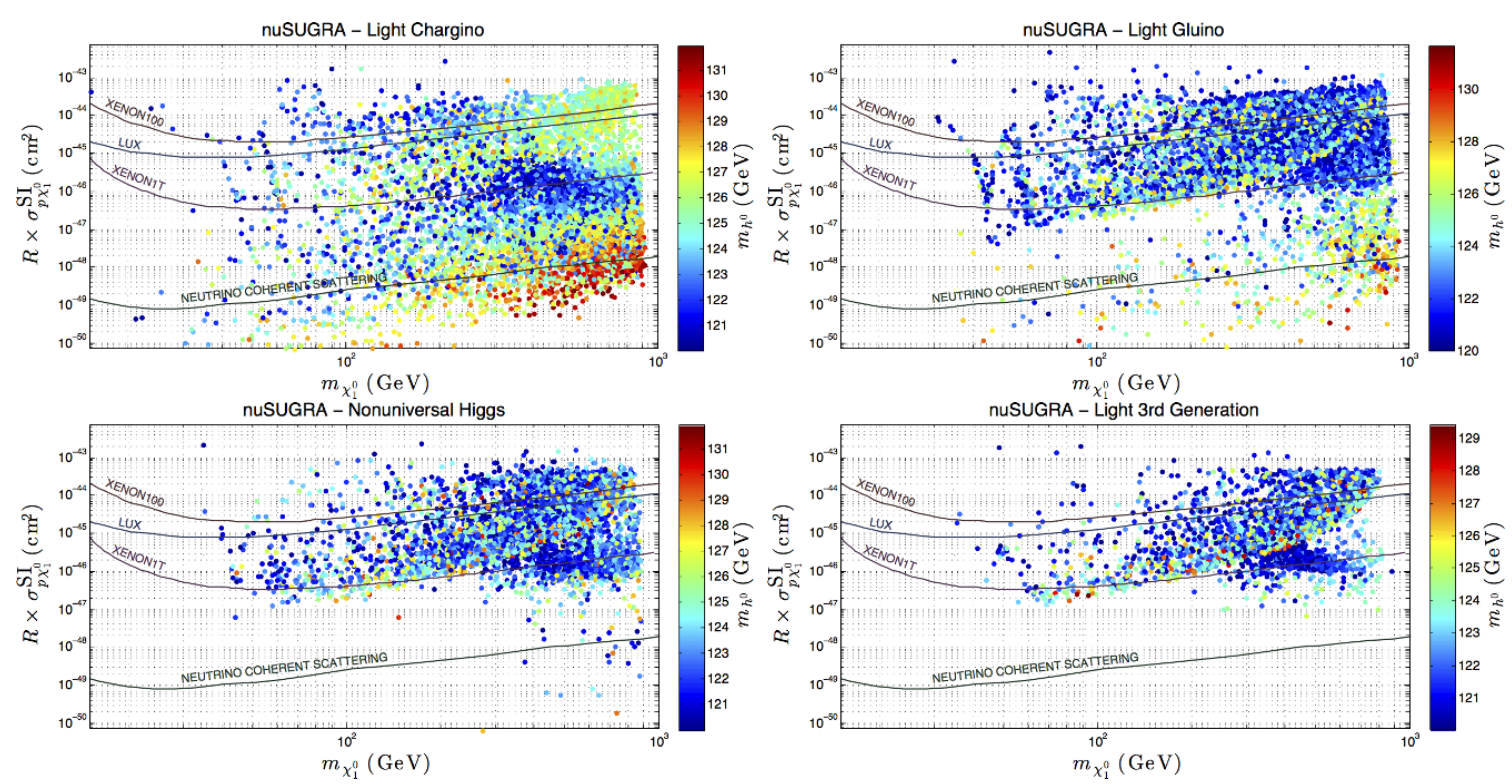

Figure 8. Top to bottom: a display of $R \times \sigma_{p \chi_{1}^{0}}^{\mathrm{SI}}$ vs the neutralino mass for nuSUGRA Models [2]Model [5]. The color specifies the mass of the Higgs boson. The cross section for neutrino-coherent scattering (the neutrino floor) is also plotted [82].

very interesting phenomenon relates to the fact that dark matter searches can be used in part as a diagnostic for the type of underlying sparticle pattern and thus of the sparticle mass hierarchy. From the right panel of figure 7 and from figure 10 and figure 11, we note that the models which dominate the parameter space mostly have chargino as NLSP. However, there are corners where some patterns are more frequent than others. Thus, for example, in the right panel of figure 7 all the models that lie close to the neutrino coherent scattering line are the ones where the stop is the NLSP and the ones above those are mostly where the lightest stau is the NLSP. Further, the region between neutralino mass of 


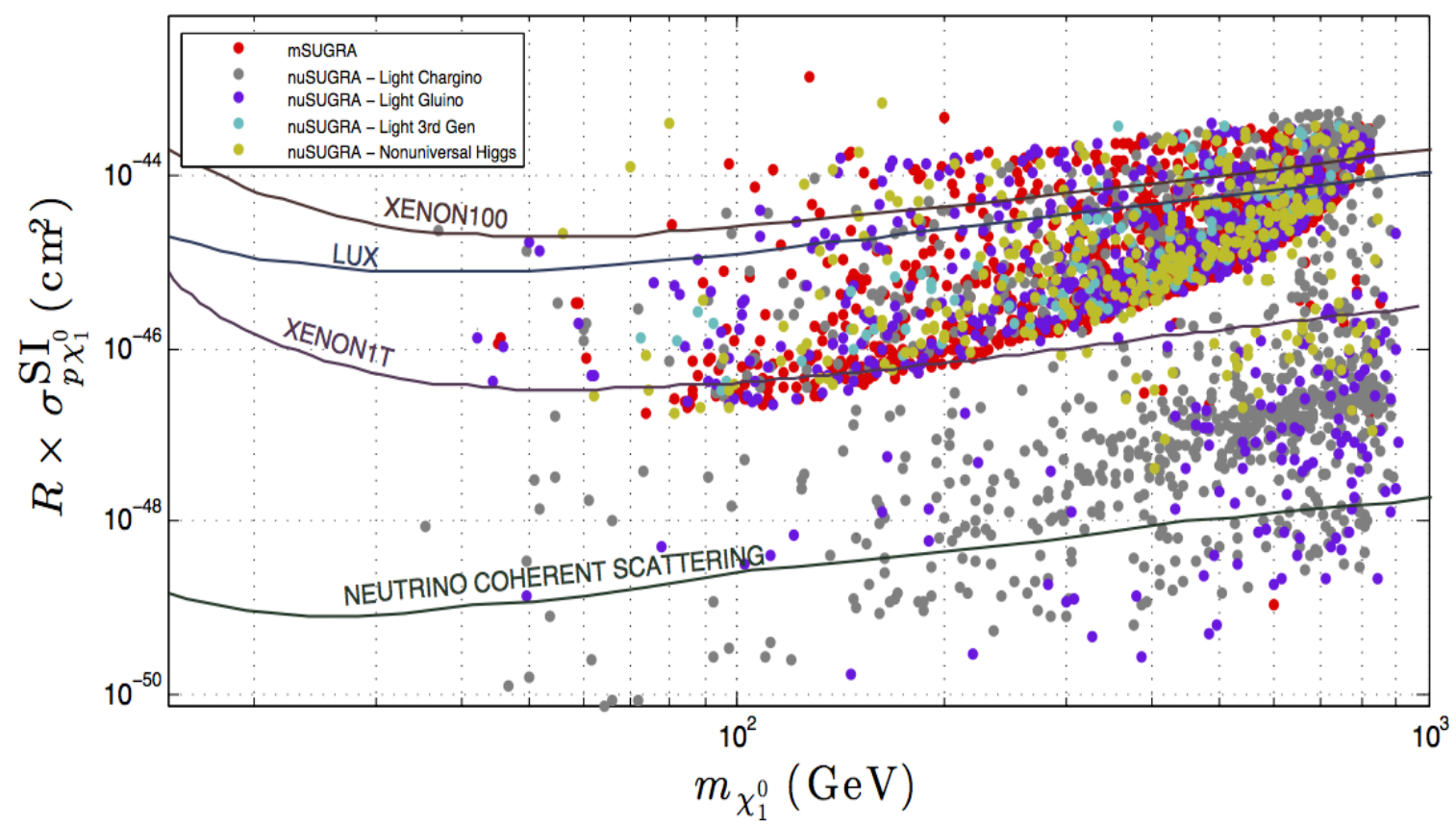

Figure 9. A display of $R \times \sigma_{p \chi_{1}^{0}}^{\mathrm{SI}}$ vs the neutralino mass which is a composite for Models [1]-[5] with the additional constraints, $m_{h^{0}} \in[123,127] \mathrm{GeV}, \Omega h^{2}<0.12, B_{s}^{0} \rightarrow \mu^{+} \mu^{-}<6.2 \times 10^{-9}, B \rightarrow$ $X_{s} \gamma<4.27 \times 10^{-4}$.
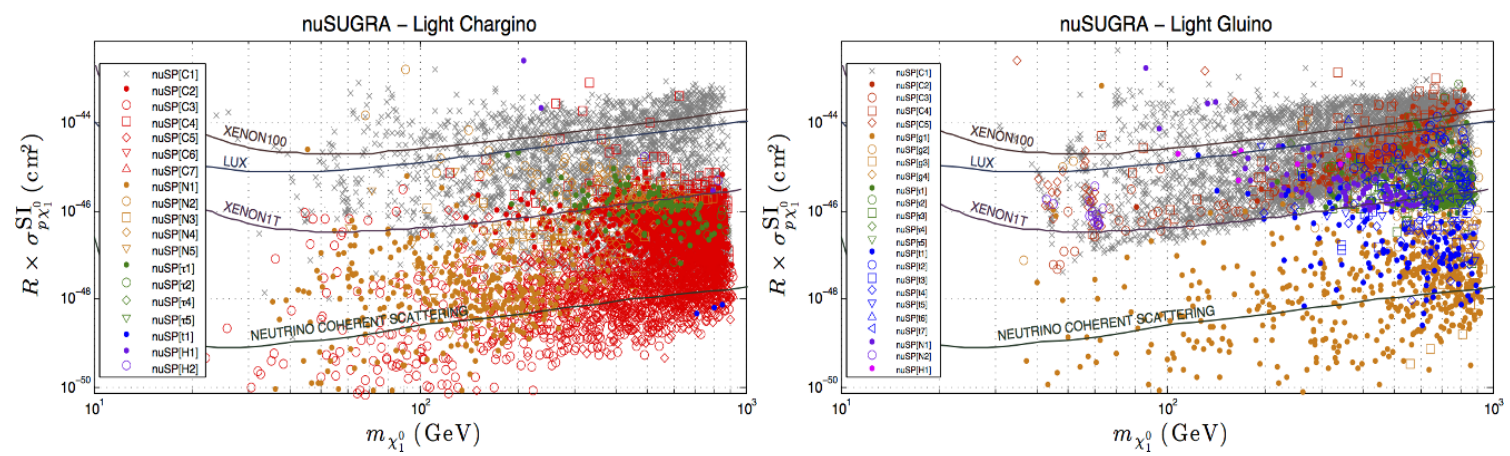

Figure 10. Dark matter plots of the nuSUGRA models, including the light chargino case (Model [2]) and the light gluino case (Model [3]). The color and shape specifies the sparticle mass pattern.

60-105 GeV which lies below the LUX limit consists exclusively of chargino NLSP patterns. In the analysis of nuSUGRA models as shown in figures 10 and 11 one finds that there are certain regions where only very specific patterns appear while in others only a combination of two or three patterns appear. In these cases a knowledge of the spin-independent cross section along with a knowledge of the neutralino mass hopefully from collider data will allow us to narrow down the possibilities for the allowed hierarchical patterns. This would help delineate the nature of high scale boundary conditions for the underlying supergravity grand unification model.

The analysis of figures 7 to 11 shows that certain sparticle patterns often give too small a cross section which are below the reach of XENON1T and other similar size dark matter 

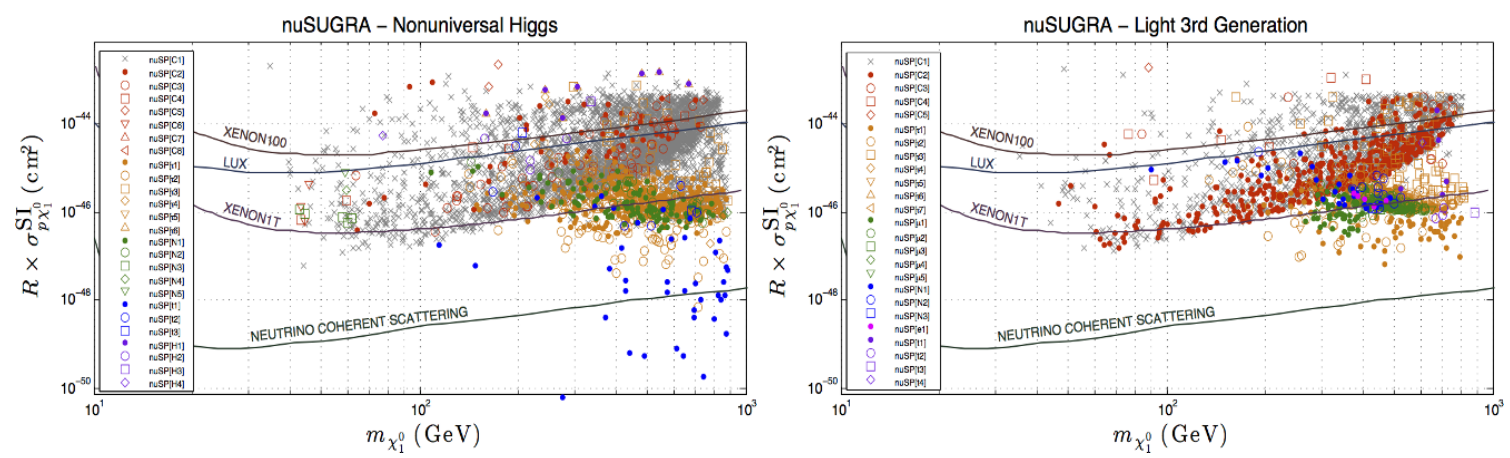

Figure 11. Dark matter plots of the nuSUGRA models, including the nonuniversal Higgs case (Model [4]) and the light third generation case (Model [5]). The color and the shape specifies the sparticle mass pattern.

experiments while some of the other patterns have cross sections which lie even below the neutrino floor. Specifically from the lower panel of figure 7 we see that the stop patterns, i.e., $\mathrm{mSP}[t 1]$ have cross sections which cross the neutrino coherent scattering line and thus the cross sections from these would be extremely challenging to observe. The reason for the extreme smallness of the cross sections in that the neutralino in these cases is essentially almost $100 \%$ bino-like, and do not have light first and second generation squark states available which suppresses the strength of interaction in the scattering off nuclei. Similar observations apply to the analysis of figures 8 to 11 . In table 11, we display the bino and Higgsino fractions of such model points along with the corresponding spin-independent neutralino-proton cross sections. To explain the smallness of the spin-independent cross section further for some of the simplified models, we consider the explicit form of the scalar cross section for the neutralino-nucleus scattering which is given by

$$
\sigma_{\chi_{1}^{0} N}^{\mathrm{SI}}=\left(4 \mu_{r}^{2} / \pi\right)\left(Z f_{p}+(A-Z) f_{n}\right)^{2} .
$$

where $Z$ is the total number of protons, $A$ the total number of protons and neutrons in the nucleus, and $\mu_{r}$ is the neutralino reduced mass. For the case when the squarks are very heavy, the s-channel pole diagrams in neutralino-quark scattering give a relatively small contribution which is dominated by the t-channel Higgs-boson exchanges. In this circumstance $f_{p / n}$ are given by

$$
f_{p / n}=\sum_{q=u, d, s} f_{T_{q}}^{(p / n)} C_{q} \frac{m_{p / n}}{m_{q}}+\frac{2}{27} f_{T G}^{(p / n)} \sum_{q=c, b, t} C_{q} \frac{m_{p / n}}{m_{q}},
$$

where the form factors $f_{T_{q}}^{(p / n)}$ and $f_{T G}^{(p / n)}$ are given in [83-85] and the couplings $C_{i}$ are given by

$$
C_{q}=-\frac{g_{2} m_{q}}{4 m_{W} \delta_{3}}\left[\left(g_{2} n_{12}-g_{Y} n_{11}\right) \delta_{1} \delta_{4} \delta_{5}\left(-\frac{1}{m_{H}^{2}}+\frac{1}{m_{h}^{2}}\right)+\left(g_{2} n_{12}-g_{Y} n_{11}\right) \delta_{2}\left(\frac{\delta_{4}^{2}}{m_{H}^{2}}+\frac{\delta_{5}^{2}}{m_{h}^{2}}\right)\right] .
$$

In the above $n_{11}$, etc are defined so that $\chi_{1}^{0}=n_{11} \tilde{B}+n_{12} \tilde{W}_{3}+n_{13} \tilde{H}_{1}+n_{14} \tilde{H}_{2}$, where $\tilde{B}, \tilde{W}_{3}, \tilde{H}_{1}, \tilde{H}_{2}$ are the bino, wino, Higgsino 1 and Higgsino 2 fields. Further, $\delta_{i}$ are defined so that for up quarks $\delta_{i}=\left(n_{13}, n_{14}, \sin \beta, \sin \alpha, \cos \alpha\right)$ and for down quarks $\delta_{i}=$ 


\begin{tabular}{|c||c|c|c|c||c|c|c|c||c|c|c|}
\hline \multicolumn{1}{|c|}{$\begin{array}{c}\text { SUGRA } \\
\text { Hierarchy }\end{array}$} & $\begin{array}{c}m_{0} \\
(\mathrm{GeV})\end{array}$ & $\begin{array}{c}m_{1 / 2} \\
(\mathrm{GeV})\end{array}$ & $\begin{array}{c}A_{0} \\
(\mathrm{GeV})\end{array}$ & $\begin{array}{c}\tan \beta \\
\left(\frac{v_{2}}{v_{1}}\right)\end{array}$ & $\delta_{M_{2}}$ & $\delta_{M_{3}}$ & $\left(\delta_{H_{1}}, \delta_{H_{2}}\right)$ & $\delta_{M_{q_{3}}}$ & $\begin{array}{c}R \times \sigma_{p, \chi_{1}^{0}}^{\mathrm{SI}} \\
\left(\mathrm{cm}^{2}\right)\end{array}$ & $\begin{array}{c}\text { LSP Bino } \\
\text { Fraction }\end{array}$ & $\begin{array}{c}\text { LSP Higgsino } \\
\text { Fraction }\end{array}$ \\
\hline $\operatorname{mSP}[H 1 \mathrm{~b}]$ & 2221 & 424 & -3862 & 56 & - & - & - & - & $3.00 \times 10^{-45}$ & 0.9997 & 0.0213 \\
$\operatorname{mSP}[H 2]$ & 3762 & 416 & -6727 & 54 & - & - & - & - & $7.76 \times 10^{-46}$ & 0.9994 & 0.0331 \\
$\operatorname{mSP}[t 1 \mathrm{a}]$ & 3075 & 1706 & 13772 & 18 & - & - & - & - & $6.33 \times 10^{-49}$ & 0.9999 & 0.0122 \\
$\left.\operatorname{mSP}_{t} t 1 \mathrm{~b}\right]$ & 4032 & 1808 & 15522 & 12 & - & - & - & - & $1.17 \times 10^{-48}$ & 0.9999 & 0.0108 \\
$\operatorname{nuSP}_{2}[N 1 \mathrm{a}]$ & 9874 & 455 & -13049 & 25 & -0.480 & - & - & - & $4.70 \times 10^{-49}$ & 0.9998 & 0.0132 \\
$\operatorname{nuSP}_{2}[N 1 \mathrm{~b}]$ & 4334 & 535 & -7758 & 10 & -0.458 & - & - & - & $5.01 \times 10^{-48}$ & 0.9996 & 0.0171 \\
$\operatorname{nuSP}_{3}[g 1 \mathrm{a}]$ & 9072 & 1963 & -11390 & 27 & - & -0.821 & - & - & $5.01 \times 10^{-48}$ & 0.9998 & 0.0184 \\
$\operatorname{nuSP}_{3}[g 1 \mathrm{~b}]$ & 6695 & 1959 & -10789 & 31 & - & -0.832 & - & - & $4.98 \times 10^{-49}$ & 0.9998 & 0.0156 \\
$\operatorname{nuSP}_{4}[t 1 \mathrm{a}]$ & 6083 & 1665 & 17544 & 12 & - & - & $(1.032,-1.692)$ & - & $9.93 \times 10^{-49}$ & 0.9999 & 0.0100 \\
$\operatorname{nuSP}_{4}[\tau 2 \mathrm{a}]$ & 3204 & 1585 & -7123 & 53 & - & - & $(-2.182,-2.439)$ & - & $3.34 \times 10^{-48}$ & 0.9999 & 0.0125 \\
$\operatorname{nuSP}_{5}[\tau 1 \mathrm{~b}]$ & 465 & 1077 & -3457 & 46 & - & - & - & 2.055 & $6.56 \times 10^{-48}$ & 0.9997 & 0.0217 \\
$\operatorname{nuSP}_{5}[\tau 2 \mathrm{~b}]$ & 504 & 601 & -2630 & 35 & - & - & - & 0.611 & $9.37 \times 10^{-48}$ & 0.9994 & 0.0334 \\
\hline
\end{tabular}

Table 11. A sample of mSUGRA and nuSUGRA model points with especially low spin-independent neutralino-proton cross section are given, accompanied by the sparticle mass pattern to which they belong. As displayed, the gaugino-Higgsino content of the LSP is almost entirely bino for these parameter points. Since these points exhibit a nearly $100 \%$ bino-like LSP, this gives reason for the smallness of the observed cross sections. The Higgsino fraction given in the last column is defined as $\sqrt{n_{13}^{2}+n_{14}^{2}}$.

$\left(n_{14},-n_{13}, \cos \beta, \cos \alpha,-\sin \alpha\right)$, where $i$ runs from 1 to 5 and $\alpha$ is the neutral Higgs mixing parameter. From eq. (6.3) we see that the cross section depends directly on $\delta_{1}, \delta_{2}$ and consequently on $n_{13}, n_{14}$ and hence on the Higgsino fraction. From the last column of table 11 we see that the Higgsino content is indeed very small for the patterns listed in table 11 which explains the smallness of spin-independent cross sections listed in the 10th column of table 11 and also explains the smallness of the cross sections that appear in figures 7 to 11 .

\section{Conclusion}

In this work we have analyzed the landscape of sparticle mass hierarchies that are consistent with the Higgs boson mass measurement. Sparticle mass hierarchies are crucial for understanding the nature of symmetry breaking at the high scale. As the soft breaking parameters at the grand unification scale will in general be nonuniversal, the different patterns of soft parameters as well as their specific values lead to a variety of different hierarchical patterns for sparticle masses. For 31 particle masses beyond the standard model one finds that a landscape of $10^{33}$ mass hierarchies arises. A large number of these are eliminated under the constraints of electroweak symmetry breaking, and collider and dark matter constraints. However, the residual number of patterns is still large. This number is drastically reduced when one considers the hierarchical patterns for the case when the number of particles taken into account is five, including the neutralino LSP.

The analysis of the hierarchical patterns is done for several different high scale models. These include [1] the mSUGRA model with universal boundary conditions, [2] the nonuni- 
versal SUGRA model with nonuniversality in the $\mathrm{SU}(2)_{L}$ gaugino mass sector, [3] the nonuniversal SUGRA model with nonuniversality in the $\mathrm{SU}(3)_{C}$ gaugino mass sector, [4] the nonuniversal SUGRA model with nonuniversality in the Higgs boson mass sector, and [5] the nonuniversal SUGRA model with nonuniversality in the third generation sfermion sector. Further, we have produced lists of simplified models with 3, 4 or 5 sparticle mass hierarchies along with their relative occurrences that arise from supergravity models with universal and nonuniversal boundary conditions. These hierarchical patterns will be helpful in establishing the nature of the high scale models which give rise to the hierarchical patterns. In addition we have provided benchmarks for SUGRA Models, which span the parameter space of supergravity models with universal as well as nonuniversal boundary conditions. These benchmarks should prove to be useful for SUSY searches at the LHC Run-II. We have also carried out an explicit signature analysis for two benchmark models, one for a supergravity model with universal boundary conditions and the other for a supergravity model with nonuniversal boundary conditions.

In addition to the analysis of sparticle mass hierarchies we have also analyzed the spin-independent neutralino-proton cross section for the five classes of supergravity Models discussed in section 2. It is found that the latest limits from the LUX dark matter experiment probe a significant part of the parameter space of models, and the XENON1T and SuperCDMS will be able to exhaust significantly more of the parameter space of many of the SUGRA models. However, for the case of the SUGRA models with nonuniversalities especially in the $\mathrm{SU}(2)_{L}$ and $\mathrm{SU}(3)_{C}$ gaugino sectors, the neutralino-proton cross sections extend downwards even past the so-called neutrino floor as shown in figures 8 to 11 . Another important aspect of the analysis relates to the diagnostic of the spin-independent cross section vs the neutralino mass. An analysis of the patterns in the spin-independent cross section vs the neutralino mass-plane one finds that certain regions of the plane are populated dominantly by one or two patterns. Thus a simultaneous measurement of the spin-independent cross section and a knowledge of the neutralino mass, such as from collider experiments, could isolate the likely sparticle mass hierarchies and thus provide strong clues to the nature of symmetry breaking in high scale models. Thus dark matter analyses along with analyses of the LHC Run-II can allow one to pin down in a concrete way the nature of the high scale models leading to the sparticle mass patterns.

\section{Acknowledgments}

Discussions with Zuowei Liu and Meenakshi Narain are acknowledged. The research of D.F. and P.N. was supported in part by NSF grants PHY-1314774 and PHY-0969739, and by XSEDE grant TG-PHY110015. The research of S.A. was supported by European Union and the European Social Fund through LHCPhenoNet network PITN-GA-2010264564. The research of B.A. was supported in part by the U.S. Department of Energy under Grant No. DE-FG02-13ER41979. This research used resources of the National Energy Research Scientific Computing Center, which is supported by the Office of Science of the U.S. Department of Energy under Contract No. DE-AC02-05CH11231, and of the OU Supercomputing Center for Education \& Research (OSCER) at the University of Oklahoma (OU). 
Open Access. This article is distributed under the terms of the Creative Commons Attribution License (CC-BY 4.0), which permits any use, distribution and reproduction in any medium, provided the original author(s) and source are credited.

\section{References}

[1] CMS collaboration, Observation of a new boson at a mass of $125 \mathrm{GeV}$ with the CMS experiment at the LHC, Phys. Lett. B 716 (2012) 30 [arXiv:1207.7235] [INSPIRE].

[2] ATLAS collaboration, Observation of a new particle in the search for the Standard Model Higgs boson with the ATLAS detector at the LHC, Phys. Lett. B 716 (2012) 1 [arXiv: 1207.7214] [INSPIRE].

[3] F. Englert and R. Brout, Broken Symmetry and the Mass of Gauge Vector Mesons, Phys. Rev. Lett. 13 (1964) 321 [INSPIRE].

[4] P.W. Higgs, Broken Symmetries and the Masses of Gauge Bosons, Phys. Rev. Lett. 13 (1964) 508 [INSPIRE].

[5] G.S. Guralnik, C.R. Hagen and T.W.B. Kibble, Global Conservation Laws and Massless Particles, Phys. Rev. Lett. 13 (1964) 585 [INSPIRE].

[6] S. Akula, B. Altunkaynak, D. Feldman, P. Nath and G. Peim, Higgs Boson Mass Predictions in SUGRA Unification, Recent LHC-7 Results and Dark Matter, Phys. Rev. D 85 (2012) 075001 [arXiv: 1112.3645] [INSPIRE].

[7] H. Baer, V. Barger and A. Mustafayev, Implications of a 125 GeV Higgs scalar for LHC SUSY and neutralino dark matter searches, Phys. Rev. D 85 (2012) 075010 [arXiv: 1112.3017] [INSPIRE].

[8] A. Arbey, M. Battaglia, A. Djouadi, F. Mahmoudi and J. Quevillon, Implications of a 125 GeV Higgs for supersymmetric models, Phys. Lett. B 708 (2012) 162 [arXiv:1112.3028] [INSPIRE].

[9] P. Draper, P. Meade, M. Reece and D. Shih, Implications of a $125 \mathrm{GeV}$ Higgs for the MSSM and Low-Scale SUSY Breaking, Phys. Rev. D 85 (2012) 095007 [arXiv:1112.3068] [INSPIRE].

[10] M. Carena, S. Gori, N.R. Shah and C.E.M. Wagner, A $125 \mathrm{GeV}$ SM-like Higgs in the MSSM and the $\gamma \gamma$ rate, JHEP 03 (2012) 014 [arXiv: 1112.3336] [INSPIRE].

[11] S. Akula, P. Nath and G. Peim, Implications of the Higgs Boson Discovery for mSUGRA, Phys. Lett. B 717 (2012) 188 [arXiv:1207.1839] [INSPIRE].

[12] A. Arbey, M. Battaglia, A. Djouadi and F. Mahmoudi, The Higgs sector of the phenomenological MSSM in the light of the Higgs boson discovery, JHEP 09 (2012) 107 [arXiv: 1207.1348] [INSPIRE].

[13] C. Strege, G. Bertone, F. Feroz, M. Fornasa, R. Ruiz de Austri and R. Trotta, Global Fits of the CMSSM and NUHM including the LHC Higgs discovery and new XENON100 constraints, JCAP 04 (2013) 013 [arXiv:1212.2636] [INSPIRE].

[14] A.H. Chamseddine, R.L. Arnowitt and P. Nath, Locally Supersymmetric Grand Unification, Phys. Rev. Lett. 49 (1982) 970 [INSPIRE].

[15] P. Nath, R.L. Arnowitt and A.H. Chamseddine, Gauge Hierarchy in Supergravity Guts, Nucl. Phys. B 227 (1983) 121 [INSPIRE]. 
[16] L.J. Hall, J.D. Lykken and S. Weinberg, Supergravity as the Messenger of Supersymmetry Breaking, Phys. Rev. D 27 (1983) 2359 [INSPIRE].

[17] R.L. Arnowitt and P. Nath, SUSY mass spectrum in SU(5) supergravity grand unification, Phys. Rev. Lett. 69 (1992) 725 [INSPIRE].

[18] L.E. Ibáñez and G.G. Ross, Supersymmetric Higgs and radiative electroweak breaking, C. R. Phys. 8 (2007) 1013 [hep-ph/0702046] [INSPIRE].

[19] O. Buchmueller et al., Higgs and Supersymmetry, Eur. Phys. J. C 72 (2012) 2020 [arXiv: 1112.3564] [INSPIRE].

[20] H. Baer, V. Barger, P. Huang, D. Mickelson, A. Mustafayev and X. Tata, Post-LHCr fine-tuning in the mSUGRA/CMSSM model with a $125 \mathrm{GeV}$ Higgs boson, Phys. Rev. D 87 (2013) 035017 [arXiv: 1210.3019] [INSPIRE].

[21] S. Akula and P. Nath, Gluino-driven radiative breaking, Higgs boson mass, muon $g-2$ and the Higgs diphoton decay in supergravity unification, Phys. Rev. D 87 (2013) 115022 [arXiv: 1304.5526] [INSPIRE].

[22] A. Avetisyan et al., Methods and Results for Standard Model Event Generation at $\sqrt{s}=14 \mathrm{TeV}, 33 \mathrm{TeV}$ and $100 \mathrm{TeV}$ Proton Colliders (A Snowmass Whitepaper), arXiv:1308.1636 [INSPIRE].

[23] D. Feldman, Z. Liu and P. Nath, The Landscape of Sparticle Mass Hierarchies and Their Signature Space at the LHC, Phys. Rev. Lett. 99 (2007) 251802 [Erratum ibid. 100 (2008) 069902] [arXiv:0707.1873] [INSPIRE].

[24] D. Feldman, Z. Liu and P. Nath, Light Higgses at the Tevatron and at the LHC and Observable Dark Matter in SUGRA and D-branes, Phys. Lett. B 662 (2008) 190 [arXiv:0711.4591] [INSPIRE].

[25] D. Feldman, Z. Liu and P. Nath, Sparticles at the LHC, JHEP 04 (2008) 054 [arXiv:0802.4085] [INSPIRE].

[26] N. Chen, D. Feldman, Z. Liu, P. Nath and G. Peim, Low Mass Gluino within the Sparticle Landscape, Implications for Dark Matter and Early Discovery Prospects at LHC-7, Phys. Rev. D 83 (2011) 035005 [arXiv: 1011.1246] [INSPIRE].

[27] C.F. Berger, J.S. Gainer, J.L. Hewett and T.G. Rizzo, Supersymmetry Without Prejudice, JHEP 02 (2009) 023 [arXiv:0812.0980] [INSPIRE].

[28] J.A. Conley, J.S. Gainer, J.L. Hewett, M.P. Le and T.G. Rizzo, Supersymmetry Without Prejudice at the LHC, Eur. Phys. J. C 71 (2011) 1697 [arXiv:1009.2539] [InSPIRE].

[29] B. Altunkaynak, B.D. Nelson, L.L. Everett, Y. Rao and I.-W. Kim, Landscape of Supersymmetric Particle Mass Hierarchies in Deflected Mirage Mediation, Eur. Phys. J. Plus 127 (2012) 2 [arXiv:1011.1439] [INSPIRE].

[30] P. Nath et al., The Hunt for New Physics at the Large Hadron Collider, Nucl. Phys. Proc. Suppl. 200-202 (2010) 185 [arXiv:1001.2693] [INSPIRE].

[31] W.-Z. Feng and P. Nath, Higgs diphoton rate and mass enhancement with vectorlike leptons and the scale of supersymmetry, Phys. Rev. D 87 (2013) 075018 [arXiv:1303.0289] [INSPIRE].

[32] N. Arkani-Hamed et al., MARMOSET: The Path from LHC Data to the New Standard Model via On-Shell Effective Theories, hep-ph/0703088 [INSPIRE]. 
[33] J. Alwall, P. Schuster and N. Toro, Simplified Models for a First Characterization of New Physics at the LHC, Phys. Rev. D 79 (2009) 075020 [arXiv:0810.3921] [InSPIRE].

[34] J. Alwall, M.-P. Le, M. Lisanti and J.G. Wacker, Model-Independent Jets plus Missing Energy Searches, Phys. Rev. D 79 (2009) 015005 [arXiv:0809.3264] [INSPIRE].

[35] D.S.M. Alves, E. Izaguirre and J.G. Wacker, Where the Sidewalk Ends: Jets and Missing Energy Search Strategies for the 7 TeV LHC, JHEP 10 (2011) 012 [arXiv:1102.5338] [INSPIRE].

[36] LHC New Physics Working Group collaboration, D.S.M. Alves et al., Simplified Models for LHC New Physics Searches, J. Phys. G 39 (2012) 105005 [arXiv:1105. 2838] [inSPIRE].

[37] M. Papucci, J.T. Ruderman and A. Weiler, Natural SUSY Endures, JHEP 09 (2012) 035 [arXiv:1110.6926] [INSPIRE].

[38] R. Mahbubani, M. Papucci, G. Perez, J.T. Ruderman and A. Weiler, Light Nondegenerate Squarks at the LHC, Phys. Rev. Lett. 110 (2013) 151804 [arXiv:1212.3328] [InSPIRE].

[39] CMS collaboration, Interpretation of Searches for Supersymmetry with simplified Models, Phys. Rev. D 88 (2013) 052017 [arXiv: 1301.2175] [inSPIRE].

[40] T. Cohen et al., SUSY Simplified Models at 14, 33 and 100 TeV Proton Colliders, JHEP 04 (2014) 117 [arXiv:1311.6480] [INSPIRE].

[41] G. Anderson, C.-h. Chen, J.F. Gunion, J.D. Lykken, T. Moroi and Y. Yamada, Motivations for and implications of nonuniversal GUT scale boundary conditions for soft SUSY breaking parameters, eConf C 960625 (1996) SUP107 [hep-ph/9609457] [INSPIRE].

[42] P. Nath and R.L. Arnowitt, Nonuniversal soft SUSY breaking and dark matter, Phys. Rev. D 56 (1997) 2820 [hep-ph/9701301] [INSPIRE].

[43] J.R. Ellis, K.A. Olive and Y. Santoso, The MSSM parameter space with nonuniversal Higgs masses, Phys. Lett. B 539 (2002) 107 [hep-ph/0204192] [INSPIRE].

[44] G. Anderson, H. Baer, C.-h. Chen and X. Tata, The Reach of Fermilab Tevatron upgrades for SU(5) supergravity models with nonuniversal gaugino masses, Phys. Rev. D 61 (2000) 095005 [hep-ph/9903370] [INSPIRE].

[45] K. Huitu, Y. Kawamura, T. Kobayashi and K. Puolamaki, Phenomenological constraints on SUSY SU(5) GUTs with nonuniversal gaugino masses, Phys. Rev. D 61 (2000) 035001 [hep-ph/9903528] [INSPIRE].

[46] A. Corsetti and P. Nath, Gaugino mass nonuniversality and dark matter in SUGRA, strings and D-brane models, Phys. Rev. D 64 (2001) 125010 [hep-ph/0003186] [InSPIRE].

[47] U. Chattopadhyay and P. Nath, $b-\tau$ unification, $g_{\mu}-2$, the $\vec{b} s+\gamma$ constraint and nonuniversalities, Phys. Rev. D 65 (2002) 075009 [hep-ph/0110341] [INSPIRE].

[48] U. Chattopadhyay, A. Corsetti and P. Nath, Supersymmetric dark matter and Yukawa unification, Phys. Rev. D 66 (2002) 035003 [hep-ph/0201001] [INSPIRE].

[49] S.P. Martin, Non-universal gaugino masses from non-singlet F-terms in non-minimal unified models, Phys. Rev. D 79 (2009) 095019 [arXiv:0903.3568] [InSPIRE].

[50] D. Feldman, Z. Liu and P. Nath, Gluino NLSP, Dark Matter via Gluino Coannihilation and LHC Signatures, Phys. Rev. D 80 (2009) 015007 [arXiv:0905.1148] [INSPIRE].

[51] I. Gogoladze, F. Nasir and Q. Shafi, Non-Universal Gaugino Masses and Natural Supersymmetry, Int. J. Mod. Phys. A 28 (2013) 1350046 [arXiv:1212.2593] [INSPIRE]. 
[52] M. Adeel Ajaib, I. Gogoladze, Q. Shafi and C.S. Un, A Predictive Yukawa Unified SO(10) Model: Higgs and Sparticle Masses, JHEP 07 (2013) 139 [arXiv:1303.6964] [INSPIRE].

[53] B. Kaufman and B.D. Nelson, Mirage Models Confront the LHC: II. Flux-Stabilized Type IIB String Theory, Phys. Rev. D 89 (2014) 085029 [arXiv:1312.6621] [INSPIRE].

[54] S. Akula, SusyKit, http://freeboson.org/software/.

[55] B.C. Allanach, SOFTSUSY: a program for calculating supersymmetric spectra, Comput. Phys. Commun. 143 (2002) 305 [hep-ph/0104145] [INSPIRE].

[56] S. Heinemeyer, W. Hollik and G. Weiglein, FeynHiggs: A Program for the calculation of the masses of the neutral CP even Higgs bosons in the MSSM, Comput. Phys. Commun. 124 (2000) 76 [hep-ph/9812320] [INSPIRE].

[57] T. Hahn, S. Heinemeyer, W. Hollik, H. Rzehak and G. Weiglein, FeynHiggs 2.7, Nucl. Phys. Proc. Suppl. 205-206 (2010) 152 [arXiv: 1007.0956] [INSPIRE].

[58] T. Hahn, S. Heinemeyer, W. Hollik, H. Rzehak and G. Weiglein, High-Precision Predictions for the Light CP-Even Higgs Boson Mass of the Minimal Supersymmetric Standard Model, Phys. Rev. Lett. 112 (2014) 141801 [arXiv:1312.4937] [INSPIRE].

[59] G. Bélanger, N.D. Christensen, A. Pukhov and A. Semenov, SLHAplus: a library for implementing extensions of the standard model, Comput. Phys. Commun. 182 (2011) 763 [arXiv: 1008.0181] [INSPIRE].

[60] A. Fowlie and M. Raidal, Prospects for constrained supersymmetry at $\sqrt{s}=33 \mathrm{TeV}$ and $\sqrt{s}=100$ TeV proton-proton super-colliders, Eur. Phys. J. C 74 (2014) 2948 [arXiv: 1402.5419] [INSPIRE].

[61] D. Kim, P. Athron, C. Balázs, B. Farmer and E. Hutchison, Bayesian naturalness of the CMSSM and CNMSSM, Phys. Rev. D 90 (2014) 055008 [arXiv:1312.4150] [INSPIRE].

[62] A. Fowlie, Is the CNMSSM more credible than the CMSSM?, Eur. Phys. J. C 74 (2014) 3105 [arXiv: 1407.7534] [INSPIRE].

[63] L. Roszkowski, E.M. Sessolo and A.J. Williams, What next for the CMSSM and the NUHM: Improved prospects for superpartner and dark matter detection, JHEP 08 (2014) 067 [arXiv: 1405.4289] [INSPIRE].

[64] P. Nath, SUSY2014: The 22nd International Conference on Supersymmetry and Unification of Fundamental Interactions, Manchester, U.K., July 20-26 2014.

[65] A.H. Chamseddine, P. Nath and R.L. Arnowitt, Experimental Signals for Supersymmetric Decays of the $W$ and $Z$ Bosons, Phys. Lett. B 129 (1983) 445 [Erratum ibid. B 132 (1983) 467] [INSPIRE].

[66] D.A. Dicus, S. Nandi and X. Tata, W Decay in Supergravity GUTs, Phys. Lett. B 129 (1983) 451 [Erratum ibid. B 145 (1984) 448] [INSPIRE].

[67] H. Baer, K. Hagiwara and X. Tata, Gauginos as a Signal for Supersymmetry at $p \bar{p}$ Colliders, Phys. Rev. D 35 (1987) 1598 [INSPIRE].

[68] P. Nath and R.L. Arnowitt, Supersymmetric Signals at the Tevatron, Mod. Phys. Lett. A 2 (1987) 331 [INSPIRE].

[69] H. Baer, V. Barger, A. Lessa and X. Tata, Discovery potential for SUSY at a high luminosity upgrade of LHC14, Phys. Rev. D 86 (2012) 117701 [arXiv:1207.4846] [INSPIRE]. 
[70] B. Altunkaynak, C. Kao and K. Yang, Unveiling the MSSM Neutral Higgs Bosons with Leptons and a Bottom Quark, arXiv:1312.3011 [INSPIRE].

[71] K.L. Chan, U. Chattopadhyay and P. Nath, Naturalness, weak scale supersymmetry and the prospect for the observation of supersymmetry at the Tevatron and at the CERN LHC, Phys. Rev. D 58 (1998) 096004 [hep-ph/9710473] [INSPIRE].

[72] U. Chattopadhyay, A. Corsetti and P. Nath, WMAP data and recent developments in supersymmetric dark matter, Phys. Atom. Nucl. 67 (2004) 1188 [hep-ph/0310228] [INSPIRE].

[73] H. Baer, C. Balázs, A. Belyaev, T. Krupovnickas and X. Tata, Updated reach of the CERN LHC and constraints from relic density, $b \rightarrow s \gamma$ and $a_{\mu}$ in the mSUGRA model, JHEP 06 (2003) 054 [hep-ph/0304303] [INSPIRE].

[74] S. Akula, M. Liu, P. Nath and G. Peim, Naturalness, Supersymmetry and Implications for LHC and Dark Matter, Phys. Lett. B 709 (2012) 192 [arXiv:1111.4589] [InSPIRE].

[75] M. Liu and P. Nath, Higgs boson mass, proton decay, naturalness and constraints of the LHC and Planck data, Phys. Rev. D 87 (2013) 095012 [arXiv:1303.7472] [inSPIRE].

[76] ATLAS collaboration, Search for direct production of charginos and neutralinos in events with three leptons and missing transverse momentum in $\sqrt{s}=8 \mathrm{TeV}$ pp collisions with the ATLAS detector, JHEP 04 (2014) 169 [arXiv: 1402.7029] [INSPIRE].

[77] B. Altunkaynak, M. Holmes, P. Nath, B.D. Nelson and G. Peim, SUSY Discovery Potential and Benchmarks for Early Runs at $\sqrt{s}=7$ TeV at the LHC, Phys. Rev. D 82 (2010) 115001 [arXiv: 1008.3423] [INSPIRE].

[78] T. Sjöstrand, S. Mrenna and P.Z. Skands, PYTHIA 6.4 Physics and Manual, JHEP 05 (2006) 026 [hep-ph/0603175] [INSPIRE].

[79] DELPHES 3 collaboration, J. de Favereau et al., DELPHES 3, A modular framework for fast simulation of a generic collider experiment, JHEP 02 (2014) 057 [arXiv:1307.6346] [INSPIRE].

[80] W. Beenakker, R. Hopker and M. Spira, PROSPINO: A Program for the production of supersymmetric particles in next-to-leading order QCD, hep-ph/9611232 [INSPIRE].

[81] ATLAS collaboration, Search for strong production of supersymmetric particles in final states with missing transverse momentum and at least three b-jets at $\sqrt{s}=8 \mathrm{TeV}$ proton-proton collisions with the ATLAS detector, JHEP 10 (2014) 024 [arXiv:1407.0600] [INSPIRE].

[82] P. Cushman et al., Working Group Report: WIMP Dark Matter Direct Detection, arXiv: 1310.8327 [INSPIRE].

[83] U. Chattopadhyay, T. Ibrahim and P. Nath, Effects of CP-violation on event rates in the direct detection of dark matter, Phys. Rev. D 60 (1999) 063505 [hep-ph/9811362] [INSPIRE].

[84] J.R. Ellis, A. Ferstl and K.A. Olive, Reevaluation of the elastic scattering of supersymmetric dark matter, Phys. Lett. B 481 (2000) 304 [hep-ph/0001005] [INSPIRE].

[85] G. Bélanger, F. Boudjema, A. Pukhov and A. Semenov, Dark matter direct detection rate in a generic model with micrOMEGAs_2.2, Comput. Phys. Commun. 180 (2009) 747 [arXiv: 0803.2360] [INSPIRE]. 